\title{
Two-Thumb or Two-Finger Technique in Infant Cardiopulmonary Resuscitation by a Single Rescuer? A Meta-Analysis with GOSH Analysis
}

\author{
Chun-Yu Chang ${ }^{1,+} \oplus$, Yueh-Tseng Hou ${ }^{2,3,+}$, Yung-Jiun Chien ${ }^{4}$, Yu-Long Chen ${ }^{2,3}$, \\ Po-Chen Lin ${ }^{2,3}$, Chien-Sheng Chen ${ }^{2,3}$ and Meng-Yu Wu ${ }^{2,3, *(1)}$ \\ 1 School of Medicine, Tzu Chi University, Hualien 970, Taiwan; paulchang1231@gmail.com \\ 2 Department of Emergency Medicine, Taipei Tzu Chi Hospital, Buddhist Tzu Chi Medical Foundation, \\ New Taipei 231, Taiwan; 98311145@gms.tcu.edu (Y.-T.H.); yulong0129@gmail.com (Y.-L.C.); \\ taipeitzuchier@gmail.com (P.-C.L.); holeyeye@yahoo.com.tw (C.-S.C.) \\ 3 Department of Emergency Medicine, School of Medicine, Tzu Chi University, Hualien 970, Taiwan \\ 4 Department of Physical Medicine and Rehabilitation, Taipei Tzu Chi Hospital, Buddhist Tzu Chi \\ Medical Foundation, New Taipei 231, Taiwan; jessica.kan.48@gmail.com \\ * Correspondence: skyshangrila@gmail.com; Tel.: +886-9-861-72752 \\ + These authors contributed equally to the work.
}

Received: 9 June 2020; Accepted: 15 July 2020; Published: 19 July 2020

\begin{abstract}
Out-of-hospital infant cardiopulmonary arrest is a fatal and uncommon event. High mortality rates and poor neurological outcomes may be improved by early cardiopulmonary resuscitation (CPR). The ongoing debate over two different infant CPR techniques, the two-thumb (TT) and the two-finger (TF) technique, has remained, especially in terms of the adequate compression depth, compression rate, and hands-off time. In this article, we searched three major databases, PubMed, EMBASE (Excerpta Medica database), and CENTRAL (Cochrane Central Register of Controlled Trials), for randomized control trials which compared the outcomes of interest between the TT and TF techniques in infant CPR. The results showed that the TT technique was associated with higher proportion of adequate compression depth (Mean difference (MD): 19.99\%; 95\%, Confidence interval (CI): 9.77 to 30.22; $p<0.01)$ than the TF technique. There was no significant difference in compression rate and hands-off time. In our conclusion, the TT technique is better in terms of adequate compression depth than the TF technique, without significant differences in compression rate and hands-off time.
\end{abstract}

Keywords: cardiopulmonary resuscitation; two fingers; two thumbs; infant; chest compression

\section{Introduction}

Out-of-hospital infant cardiopulmonary arrest is a fatal and uncommon event with a high mortality rate and poor neurological outcome [1-3]. The common etiology of cardiac arrest in infants is asphyxia. Early cardiopulmonary resuscitation with effective chest compressions and rescue ventilation may improve the clinical outcome. Current guidelines for infant cardiopulmonary resuscitation (CPR) recommend two chest compression techniques: the two-finger (TF) for a single rescuer and the two-thumb encircling (TT) chest compression for two or more rescuers [4,5]. In previous literature, the TT technique provided better chest compression depth than the TF in animals and manikin models [6-9]. However, there is a concern that the TT technique may elicit this advantage at the cost of longer time in switching from compression to ventilation during CPR, especially in a single rescuer. Although there is a lack of strong evidence to confirm this concern, the TT technique is currently not recommended for a lone rescuer. In our meta-analysis, we try to confirm this hypothesis by analyzing three major factors, 
namely chest compression rate, proportion of adequate compression depth, and hands-off time, to provide strong evidence for a difference between the two chest compression techniques in infant CPR performed by a single rescuer.

\section{Method}

\subsection{Study Design}

This systematic review and meta-analysis aimed to evaluate the effects of two different CPR techniques, the two-thumb technique and the two-finger technique, on infant manikin models. This study complies with the recommendations made by the Preferred Reporting Items for Systematic Review and Meta-analysis (PRISMA) statement [10].

\subsection{Search Strategy}

Two authors (Yung-Jiun Chien and Chun-Yu Chang) searched PubMed, EMBASE, and CENTRAL. Subject headings from PubMed, CENTRAL, and EMBASE (Mesh terms and Emtree terms) were used in combination with the title and abstract field tag or free-text words to facilitate searching. The following terms were used for searching: "cardiopulmonary resuscitation", "heart arrest", "heart massage", "chest compression", "infant", "newborn", "neonate", "two-thumb", "two-finger", "two-thumb chest compression", "two-finger chest compression", "infant chest compression", "newborn chest compression", "infant cardiopulmonary resuscitation", "newborn cardiopulmonary resuscitation", "manikin", and "mannequin". We did not exclude studies by languages or geographical regions. Identified records were screened by titles, abstracts, and keywords. The reference lists of the identified records were used to manually search for relevant studies.

\subsection{Eligibility Criteria and Risk of Bias in Individual Studies}

All studies identified from electronic databases were screened and selected by two authors (Yung-Jiun Chien and Chun-Yu Chang) independently according to the inclusion criteria, with all of the following being met: (a) Randomized controlled trial (RCTs), either of parallel or crossover design; (b) comparison of conventional TT (with hands encircling the thorax) with TF; (c) studies reporting the outcomes of chest compression rate, proportion of adequate compression depth, and hands-off time; and (d) outcomes with sufficient information for meta-analysis. Two authors (Yung-Jiun Chien and Chun-Yu Chang) evaluated the methodological quality of all included studies by using the Risk of Bias 2 tool [11] for both the individually randomized, parallel-group trials and individually randomized, crossover trials. The third author (MYW) provided the consensus or discussion in the case of disagreements.

\subsection{Data Extraction and Statistical Analysis}

The relevant information was extracted by two authors (Yung-Jiun Chien and Chun-Yu Chang), including authors' names, publication year, country, study design, number of participants, the expertise of participants, CPR duration, manikin model, manikin placement, and effect estimates. The effect estimates in each included study were calculated as mean difference (MD) and standard error (SE). The summary measurement (either MD or Hedges' g, where suitable) with the 95\% CI was then derived from pooling the effect of each included study using the inverse variance method with a random-effects model (DerSimonian-Laird estimator [12]). Heterogeneity (GOSH) was assessed by the Cochran $Q$ statistic and quantified with the $I^{2}$ statistic. Subgroup analysis was performed to evaluate whether the prespecified factors could account for the heterogeneity (i.e., locale, ventilation protocol, manikin model, expertise of the participants, and the placement of the manikin). Sensitivity analysis was used to test the robustness of the results. First, a leave-one-out analysis was performed by omitting one study at a time and reperforming meta-analysis to evaluate if the leave-one-out pooled summary measurement falls outside of the $95 \%$ CI of the overall summary measurement. Second, 
in the outcomes containing studies where the data input involved the assumption of correlation, we replaced the originally assumed correlation (i.e., the lowest observed) with the highest observed one among the other studies and zero and then reperformed meta-analysis. Third, a graphical display of study heterogeneity plot was generated [13], and three unsupervised learning algorithms, i.e., k-means clustering [14], density-based spatial clustering of applications with noise (DBSCAN) [15] and Gaussian mixture models [16], were used to identify the potential outliers. Alternatively, a Baujat plot was plotted to assist in identifying potential outlier(s) by visualizing the studies located at the right side of the plot that contribute considerably to the heterogeneity and/or summary measurement [17]. Meta-analysis was reperformed after excluding the potential outliers.

\section{Results}

\subsection{Study Identification and Selection}

After searching three databases, including PubMed $(n=101)$, EMBASE $(n=359)$, and CENTRAL $(n=86), 546$ articles were identified. A total of 159 articles were duplicates. The remaining studies were screened for eligibility; then, 353 articles were excluded due to not matching inclusion criteria. A total of 34 studies were assessed with full-text review; then, 21 studies were excluded due to not reporting outcomes of interest. Finally, 13 studies were included for meta-analysis. The detailed PRISMA flow diagram is shown in Figure 1.

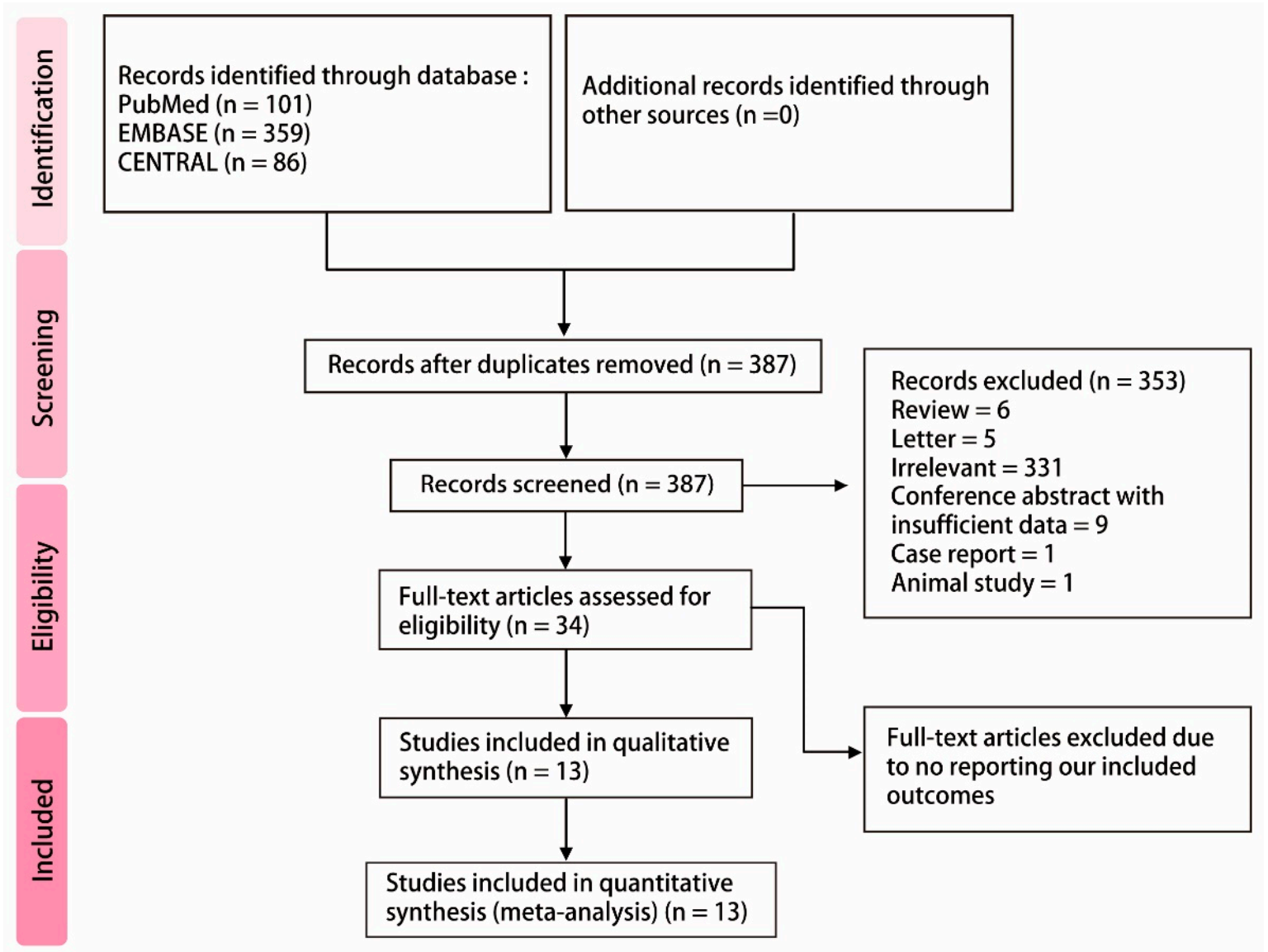

Figure 1. PRISMA flow diagram.

\subsection{Study Characteristics and Quality and Risk of Bias Assessment}

Ten studies were crossover RCTs [18-27], whereas three were parallel RCTs [28-30]. In Haque et al.'s study [30], a total of 80 participants were randomly allocated to five groups, namely the infant TF, infant TT, child one-hand, child two-hand, and adolescent two-hand groups, with 16 
participants in each group. Participants in each group were further randomized into two sequences, each starting with the compression-to-ventilation $(\mathrm{C}: \mathrm{V})$ ratio of 30:2 or 15:2. We extracted the relevant data in the infant TF and infant TT groups with C:V ratio 15:2 only. Hence, Haque et al.'s study should be regarded as parallel RCT in the present study. All studies compared the conventional TT technique to the TF technique. Participants were asked to stand at the head position while performing the TT technique (over-the-head TT) in two studies [20,21]. The CPR duration ranged from 1 to 5 min. Participants were asked to perform ventilation with the C:V ratio 30:2 in six studies and 15:2 in two studies, whereas the rest of the studies did not require the participants to perform ventilation. Participants with multiple areas of expertise were recruited in most of the studies, except for four studies where participants with a single area of expertise were recruited [19-22]. The risk of bias was assessed for each outcome, and the summary is available in Figures 2-4 and Table 1.

\begin{tabular}{|c|c|c|c|c|c|c|c|}
\hline \multirow[b]{2}{*}{ Study } & \multirow{2}{*}{$\begin{array}{l}\text { Study } \\
\text { design }\end{array}$} & \multicolumn{6}{|c|}{ RISK OF BIAS } \\
\hline & & $\begin{array}{l}\text { Bias arising from the } \\
\text { randomization process }\end{array}$ & $\begin{array}{l}\text { Bias due to deviations from } \\
\text { intended interventions }\end{array}$ & $\begin{array}{l}\text { Bias due to missing } \\
\text { outcome data }\end{array}$ & $\begin{array}{l}\text { Bias in measurement } \\
\text { of the outcome }\end{array}$ & $\begin{array}{l}\text { Bias in selection of } \\
\text { the reported result }\end{array}$ & Overall bias \\
\hline Tsou et al., 2019 & Crossover & Some concerns & Low & Low & Low & Low & Some concerns \\
\hline Lee et al., 2018 & Crossover & Some concerns & Low & Low & Low & Low & Some concerns \\
\hline Jo et al., 2017 & Crossover & Some concerns & Low & Low & Low & Low & Some concerns \\
\hline Jo et al., 2015 & Crossover & Some concerns & Low & Low & Low & Low & Some concerns \\
\hline Martin et al., 2013-A & Crossover & Some concerns & Low & Low & Low & Low & Some concerns \\
\hline Martin et al., 2013-B & Crossover & Some concerns & Low & Low & Low & Low & Some concerns \\
\hline Martin et al., 2013-C & Crossover & Some concerns & Low & Low & Low & Low & Some concerns \\
\hline Christman et al., 2011 & Crossover & Some concerns & Low & Low & Low & Low & Some concerns \\
\hline Fakhraddin et al., 2011 & Parallel & Some concerns & Low & Low & Low & Low & Some concerns \\
\hline Udassi et al., 2010 & Crossover & Some concerns & Low & Low & Low & Low & Some concerns \\
\hline Udassi et al., 2009 & Parallel & High & Low & Low & Low & Low & High \\
\hline Haque et al., 2008 & Parallel & Some concerns & Low & Low & Low & Low & Some concerns \\
\hline
\end{tabular}

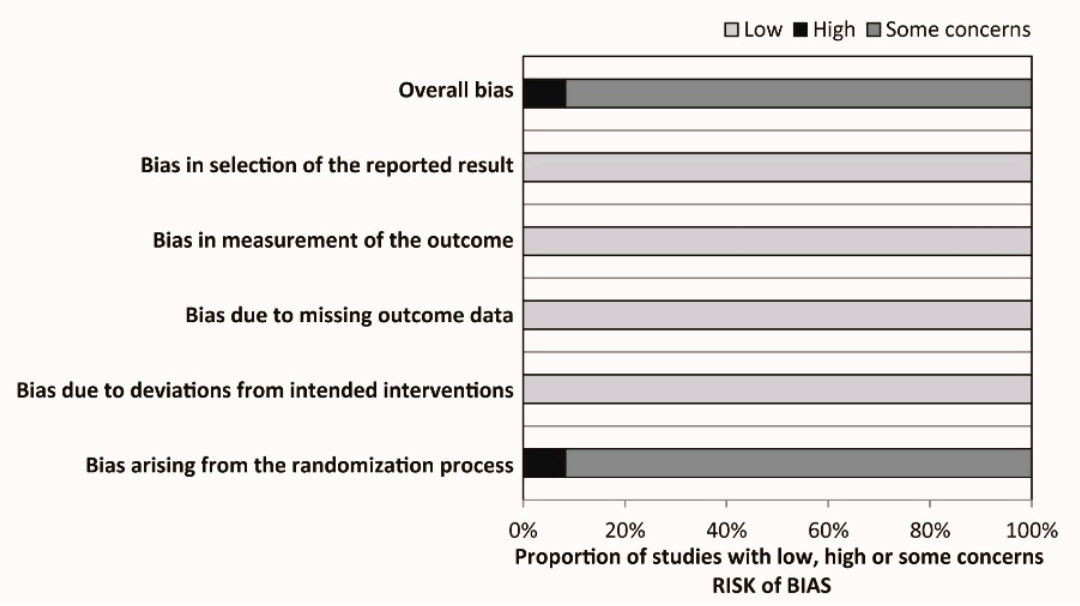

Figure 2. Risk of bias summary and graph of chest compression rate. 


\begin{tabular}{|c|c|c|c|c|c|c|c|}
\hline \multirow[b]{2}{*}{ Study } & \multirow{2}{*}{$\begin{array}{l}\text { Study } \\
\text { design }\end{array}$} & \multicolumn{6}{|c|}{ RISK OF BIAS } \\
\hline & & $\begin{array}{l}\text { Bias arising from the } \\
\text { randomization process }\end{array}$ & $\begin{array}{l}\text { Bias due to deviations from } \\
\text { intended interventions }\end{array}$ & $\begin{array}{l}\text { Bias due to missing } \\
\text { outcome data }\end{array}$ & $\begin{array}{l}\text { Bias in measurement } \\
\text { of the outcome }\end{array}$ & $\begin{array}{l}\text { Bias in selection of } \\
\text { the reported result }\end{array}$ & Overall bias \\
\hline Lee et al., 2018 & Crossover & Some concerns & Low & Low & Low & Low & Some concerns \\
\hline Jo et al., 2017 & Crossover & Some concerns & Low & Low & Low & Low & Some concerns \\
\hline Jiang et al., 2015 & Crossover & Some concerns & Low & Low & Low & Low & Some concerns \\
\hline Martin et al., 2013-B & Crossover & Some concerns & Low & Low & Low & Low & Some concerns \\
\hline Fakhraddin et al., 2011 & Parallel & Some concerns & Low & Low & Low & Low & Some concerns \\
\hline
\end{tabular}

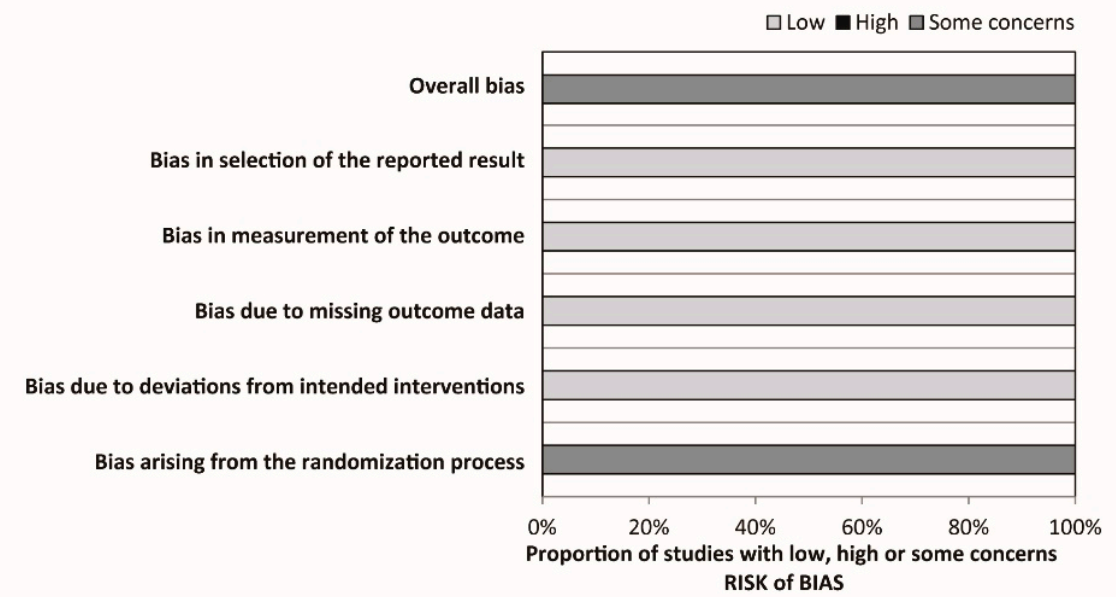

Figure 3. Risk of bias summary and graph of proportion of adequate compression depth.

\begin{tabular}{|c|c|c|c|c|c|c|c|}
\hline \multirow[b]{2}{*}{ Study } & \multirow{2}{*}{$\begin{array}{l}\text { Study } \\
\text { design }\end{array}$} & \multicolumn{6}{|c|}{ RISK OF BIAS } \\
\hline & & $\begin{array}{l}\text { Bias arising from the } \\
\text { randomization process }\end{array}$ & $\begin{array}{l}\text { Bias due to deviations from } \\
\text { intended interventions }\end{array}$ & $\begin{array}{l}\text { Bias due to missing } \\
\text { outcome data }\end{array}$ & $\begin{array}{l}\text { Bias in measurement } \\
\text { of the outcome }\end{array}$ & $\begin{array}{l}\text { Bias in selection of } \\
\text { the reported result }\end{array}$ & Overall bias \\
\hline Lee et al., 2018 & Crossover & Some concerns & Low & Low & Low & Low & Some concerns \\
\hline Jo et al., 2017 & Crossover & Some concerns & Low & Low & Low & Low & Some concerns \\
\hline Jo et al., 2015 & Crossover & Some concerns & Low & Low & Low & Low & Some concerns \\
\hline
\end{tabular}

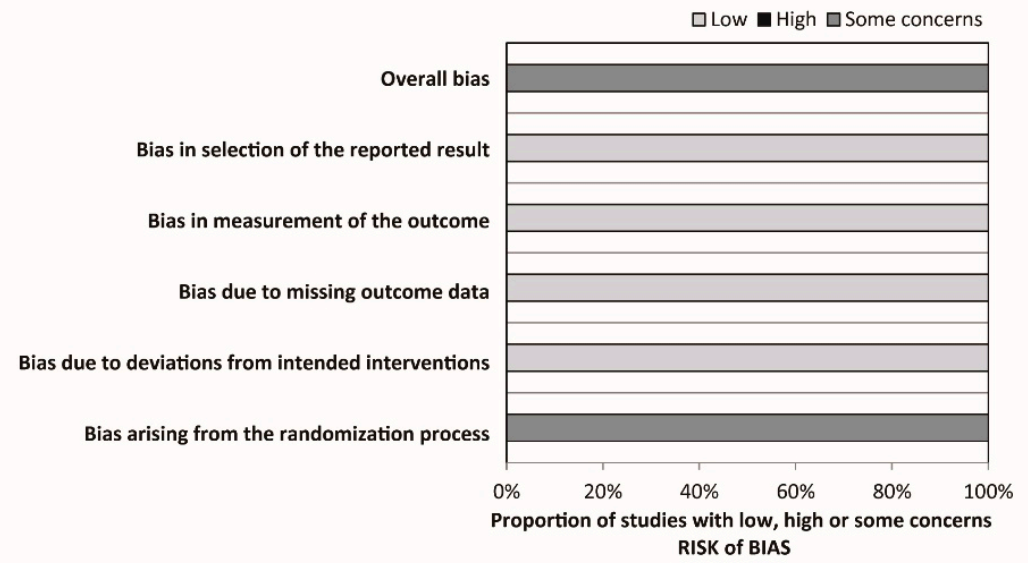

Figure 4. Risk of bias summary and graph of hands-off time. 
Table 1. The detailed characteristics of the included studies.

\begin{tabular}{|c|c|c|c|c|c|c|c|c|c|}
\hline Study & RCT Design & $\begin{array}{c}\text { Patient } \\
\text { Number }\end{array}$ & Intervention & Comparison & $\begin{array}{l}\text { CPR Time } \\
\text { (min) }\end{array}$ & Ventilation & Manikin & $\begin{array}{c}\text { Manikin } \\
\text { Placement }\end{array}$ & Participants \\
\hline Tsou et al., 2019 [18] & crossover & 42 & TT & TF & 2 & $30: 2$ & Resusci Baby QCPR (Laerdal) & $\begin{array}{c}\text { Not } \\
\text { mentioned }\end{array}$ & EMTs, RNs \\
\hline Lee et al., 2018 [19] & crossover & 37 & TT & TF & 2 & $15: 2$ & Resusci Baby QCPR (Laerdal) & Floor & Physicians \\
\hline Jo et al., 2017 [20] & crossover & 48 & OTTT & TF & 2 & $30: 2$ & Resusci Baby QCPR (Laerdal) & Bed & Medical students \\
\hline Jiang et al., 2015 [22] & crossover & 27 & TT & TF & 5 & $30: 2$ & Resusci Baby QCPR (Laerdal) & Iliac crest & Physicians \\
\hline Jo et al., 2015 [21] & crossover & 46 & OTTT & TF & 2 & $30: 2$ & Resusci Baby QCPR (Laerdal) & Bed & RNs \\
\hline Martin et al., 2013-A [24] & crossover & 22 & TT & TF & 2 & No & The Laerdal ALS Baby Trainer & Table & $\begin{array}{c}\text { Physicians, RNs, resuscitation } \\
\text { officers }\end{array}$ \\
\hline Martin et al., 2013-B [23] & crossover & 40 & TT & $\mathrm{TF}$ & 1.5 & No & The Laerdal ALS Baby Trainer & Table & $\begin{array}{l}\text { Resuscitation officer, physicians, } \\
\text { RNs, operating room practitioner, } \\
\text { paramedics }\end{array}$ \\
\hline Martin et al., 2013-C [25] & crossover & 35 & TT & TF & 1 & No & The Laerdal ALS Baby Trainer & $\begin{array}{c}\text { Not } \\
\text { mentioned }\end{array}$ & $\begin{array}{c}\text { Resuscitation officers, physicians, } \\
\text { RNs }\end{array}$ \\
\hline Christman et al., 2011 [26] & crossover & 25 & TT & $\mathrm{TF}$ & 1 & No & Laerdal HeartCode BLS manikin & $\begin{array}{c}\text { Not } \\
\text { mentioned }\end{array}$ & Physicians, RNs \\
\hline Fakhraddin et al., 2011 [28] & parallel & 40 & TT & TF & 5 & No & Resusci Baby QCPR (Laerdal) & $\begin{array}{c}\text { Not } \\
\text { mentioned }\end{array}$ & PALS providers \\
\hline Udassi et al., 2010 [27] & crossover & 34 & TT & TF & 2 & $30: 2$ & The Laerdal ALS Baby Trainer & Iliac crest & $\begin{array}{c}\text { Faculty, physicians, RNs, } \\
\text { medical/nursing students, } \\
\text { pharmacists, RTs, NPs }\end{array}$ \\
\hline Udassi et al., 2009 [29] & parallel & 32 & TT & TF & 5 & $30: 2$ & Resusci Baby QCPR (Laerdal) & Iliac crest & $\begin{array}{l}\text { RNs, medical students, physicians, } \\
\text { faculty, others }\end{array}$ \\
\hline Haque et al., 2008 [30] & parallel & 32 & TT & TF & 5 & $15: 2$ & The Laerdal ALS Baby Trainer & Iliac crest & $\begin{array}{c}\text { Faculty, physicians, RNs, } \\
\text { medical/nursing students, RTs, OTs }\end{array}$ \\
\hline
\end{tabular}

TT: two-thumb technique; OTTT: over-the-head two-thumb technique; TF: two-finger technique; CPR: cardiopulmonary resuscitation; EMT: emergency medical technician, RN: registered nurse; NP: nurse practitioner; PALS: pediatric advanced life support; RT: respiratory therapist; OT: occupational therapist. 


\subsection{Overall Summary Measurement}

There was no statistically significant difference in terms of chest compression rate (MD: $-1.05 / \mathrm{min}$; $95 \%$ CI: -3.04 to $0.93 ; p=0.30$ ). The proportion of adequate compression depth is higher using the TT technique than using the TF technique (MD: $19.99 \%$; $95 \%$ CI: 9.77 to $30.22 ; p<0.01$ ). In addition, there was no statistically significant difference in terms of the hands-off time (Hedges' g: $0.07 ; 95 \%$ CI: -0.37 to $0.51 ; p=0.76$; Figure 5).

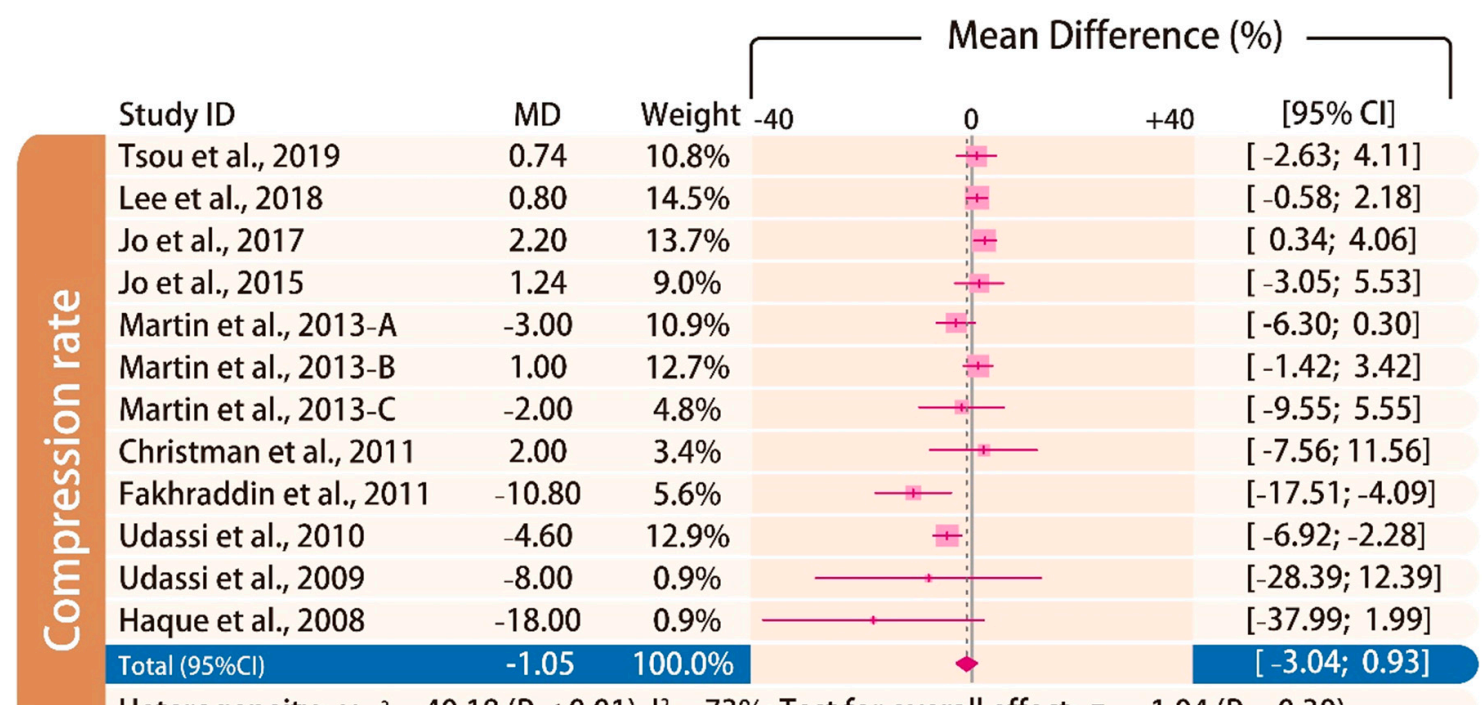

Heterogeneity: $X_{11}{ }^{2}=40.18(P<0.01), I^{2}=73 \%$; Test for overall effect: $z=-1.04(P=0.30)$

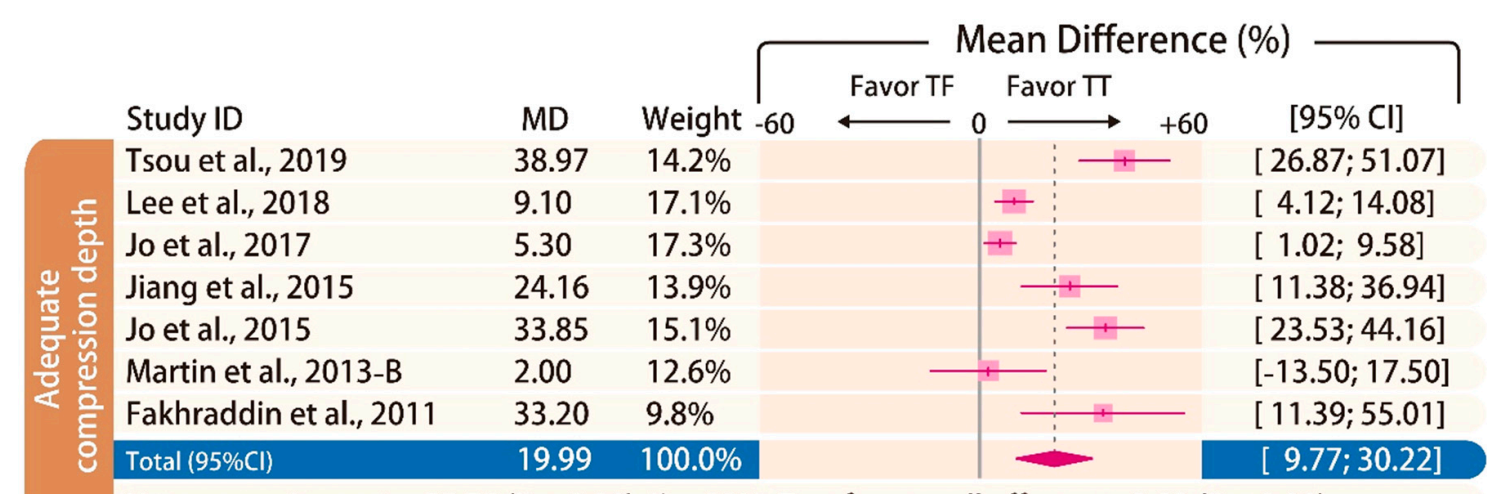

Heterogeneity: $X_{6}{ }^{2}=55.75(P<0.01), I^{2}=89 \%$; Test for overall effect: $z=3.83(P<0.01)$

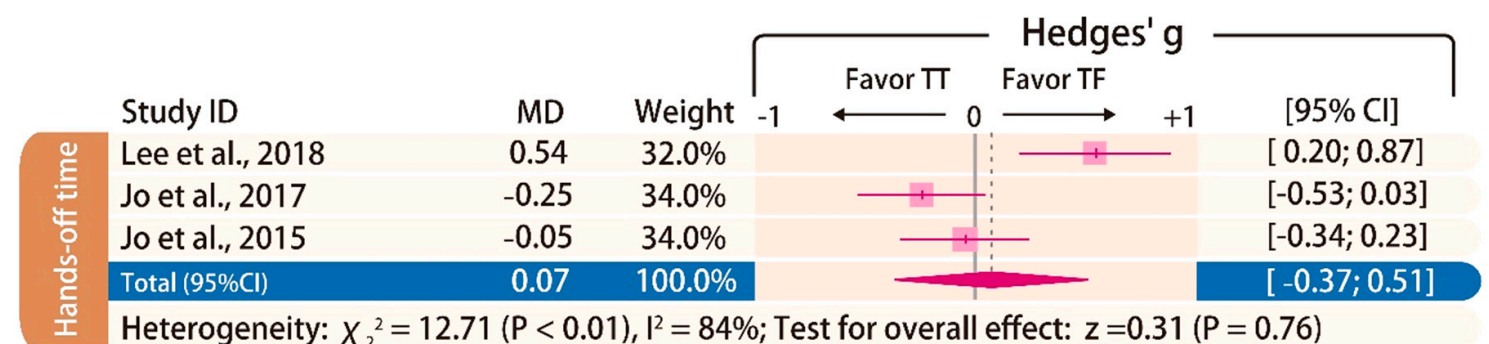

Figure 5. Forest plot of chest compression rate, proportion of adequate compression depth, and hand-off time.

\subsection{Subgroup Analysis of Chest Compression Rate}

We found that the prespecified factors could not explain the heterogeneity observed in chest compression rate. First, $\mathrm{I}^{2}$ was $71 \%$ for studies conducted in Asia, $48 \%$ for those conducted in Europe, and $18 \%$ for those conducted in North America. Second, $\mathrm{I}^{2}$ was $68 \%$ for studies that did not require the 
participants to perform ventilation and $77 \%$ for those that did. When the latter was further grouped by the $C: V$ ratio, heterogeneity remained high in both the $15: 2$ group $\left(\mathrm{I}^{2}=70 \%\right)$ and the 30:2 group $\left(\mathrm{I}^{2}=81 \%\right.$ ). Third, $\mathrm{I}^{2}$ was $71 \%$ and $64 \%$ for studies using Laerdal Resusci Baby QCPR and The Laerdal ALS Baby Trainer, respectively. Fourth, studies enrolling participants from single expertise showed low heterogeneity $\left(\mathrm{I}^{2}=0 \%\right)$, but not those with multiple areas of expertise $\left(\mathrm{I}^{2}=59 \%\right)$. Finally, studies where the manikin was placed on the bed $\left(\mathrm{I}^{2}=0 \%\right)$ and height adjusted to the iliac crest $\left(\mathrm{I}^{2}=0 \%\right)$ showed low heterogeneity but studies with the manikin on the table $\left(\mathrm{I}^{2}=73 \%\right)$ did not. The detailed results can be seen in Figures 6-11.

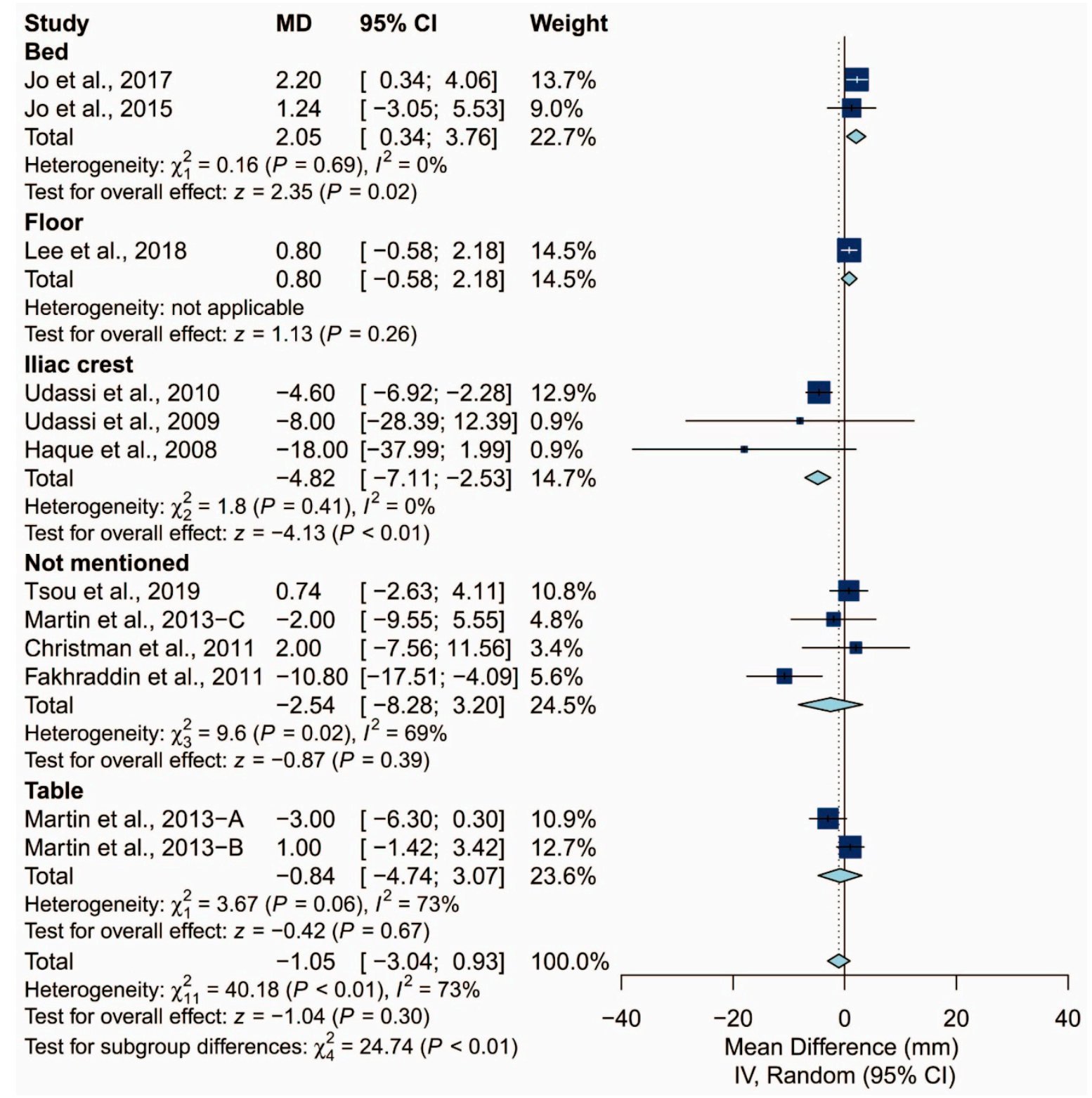

Figure 6. Subgroup analysis of chest compression rate grouped by locale. 


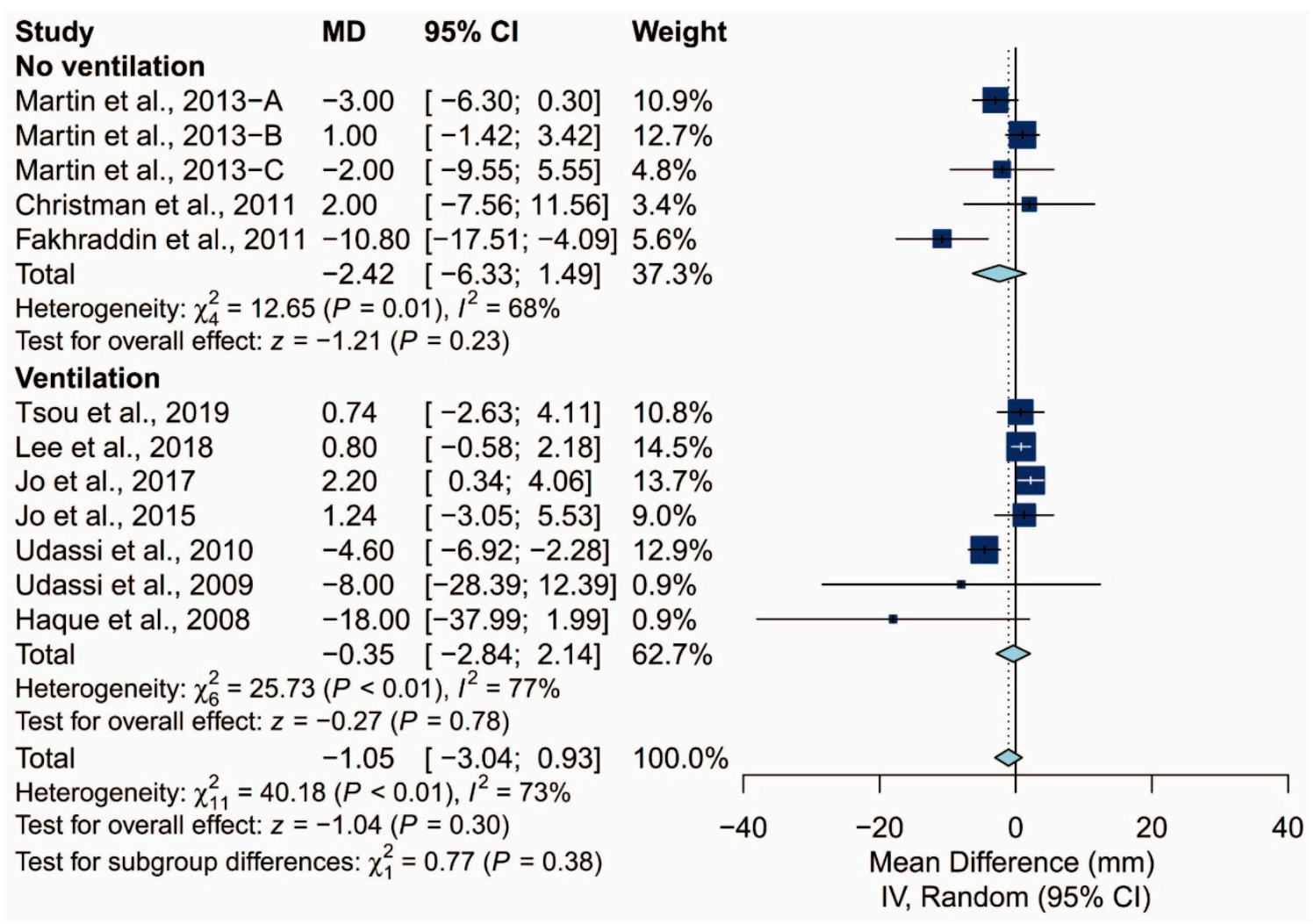

Figure 7. Subgroup analysis of chest compression rate grouped by ventilation protocol (yes/no).

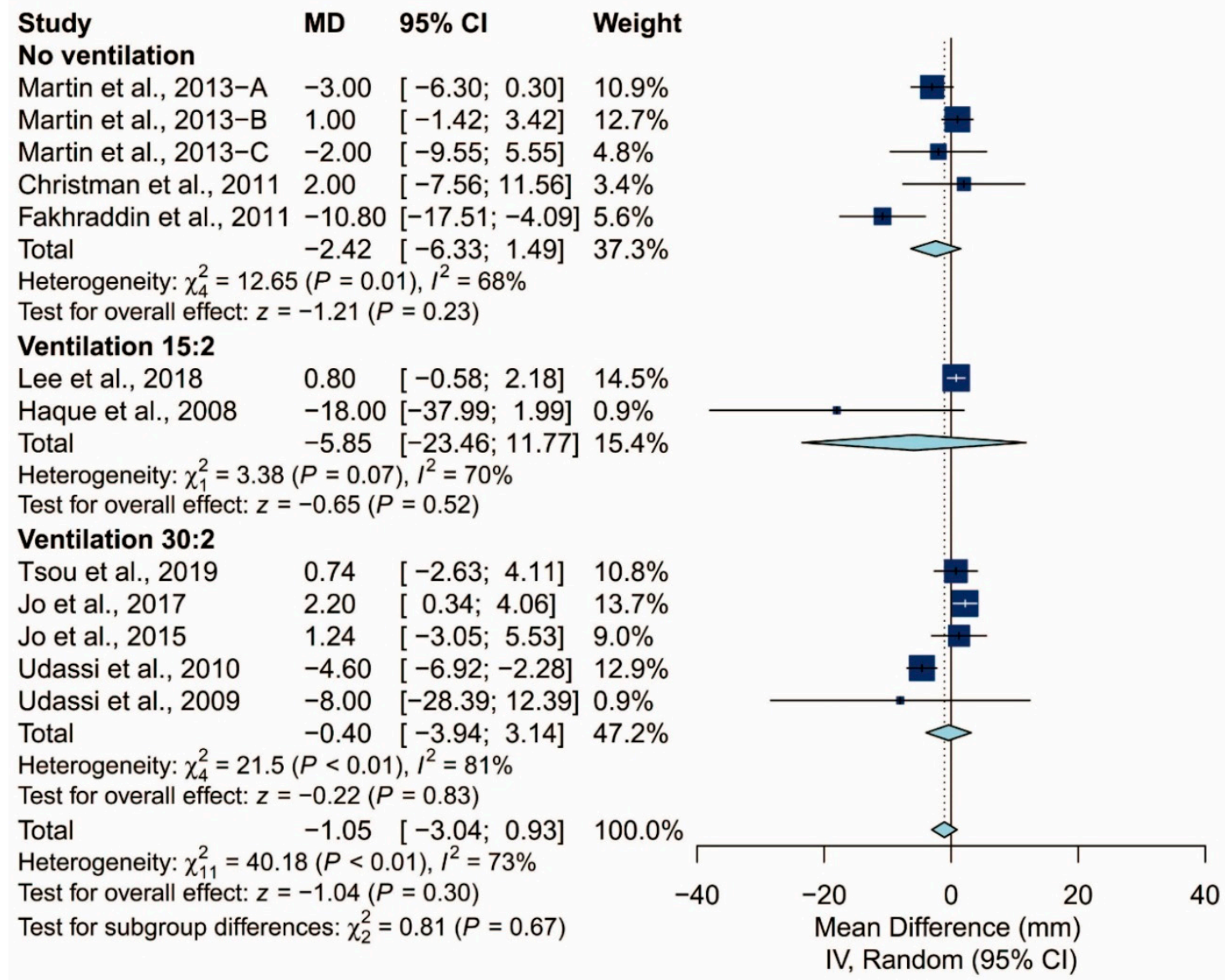

Figure 8. Subgroup analysis of chest compression rate grouped by ventilation protocol (15:2/30:2/no). 


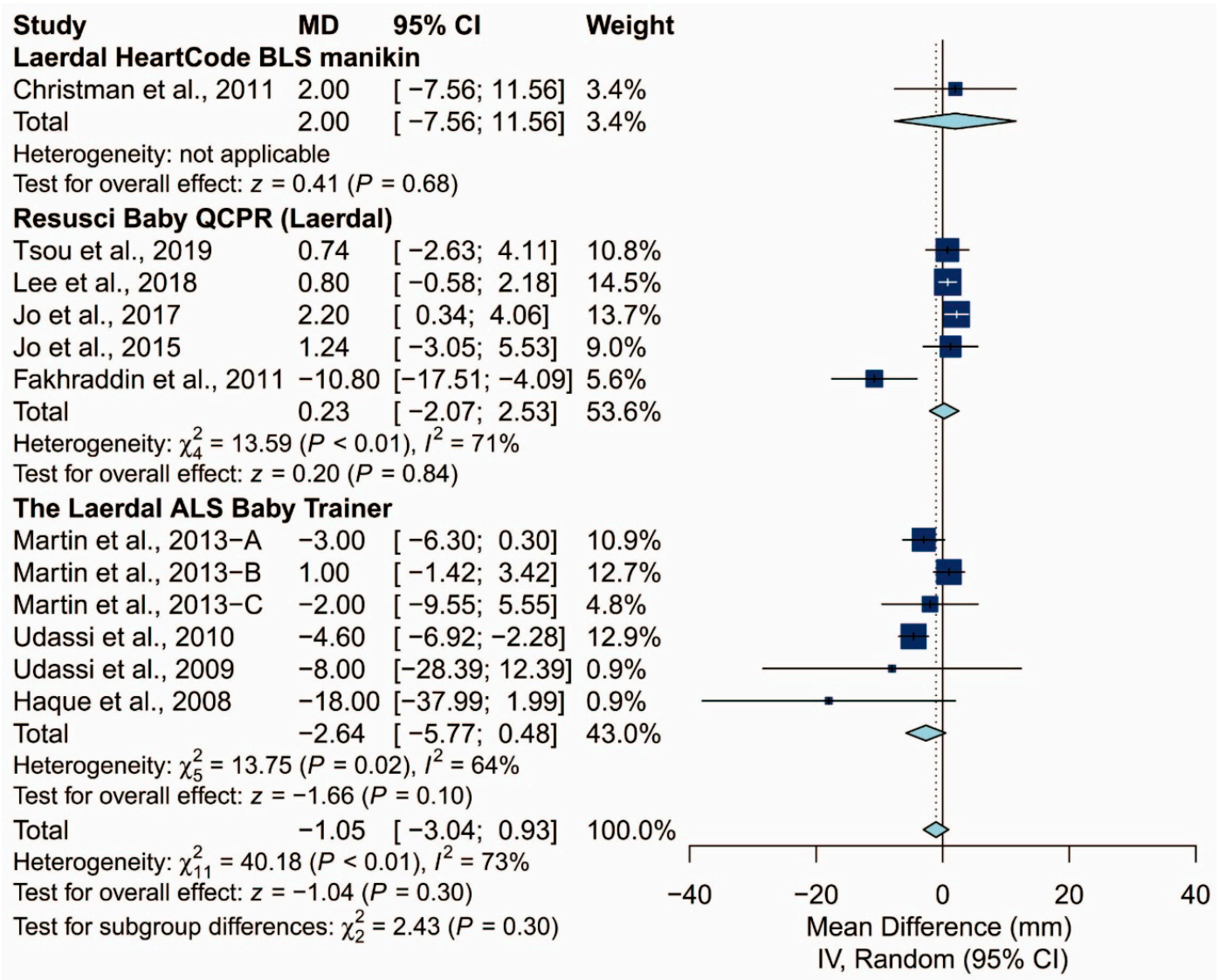

Figure 9. Subgroup analysis of chest compression rate grouped by manikin model.

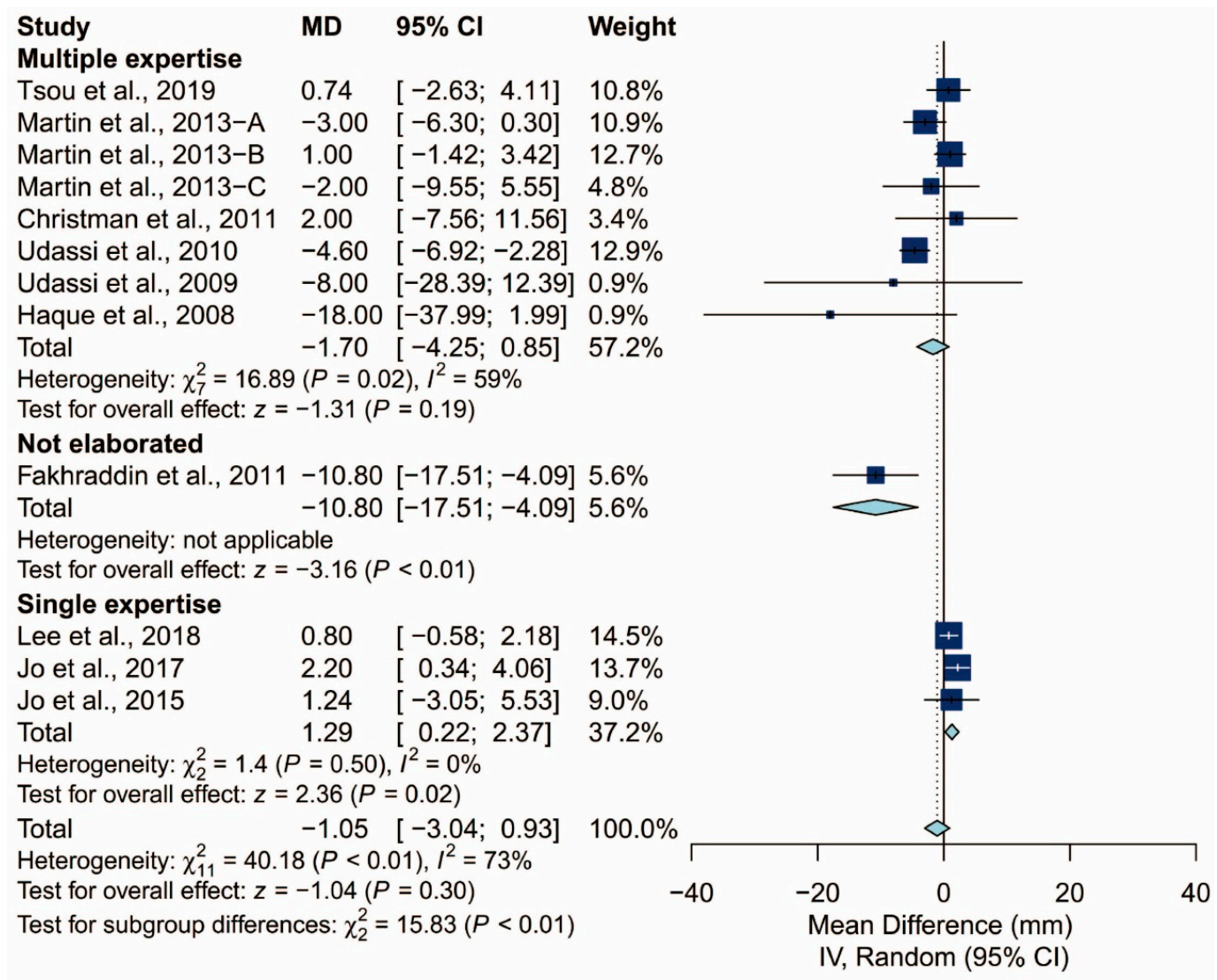

Figure 10. Subgroup analysis of chest compression rate grouped by expertise of the participants. 


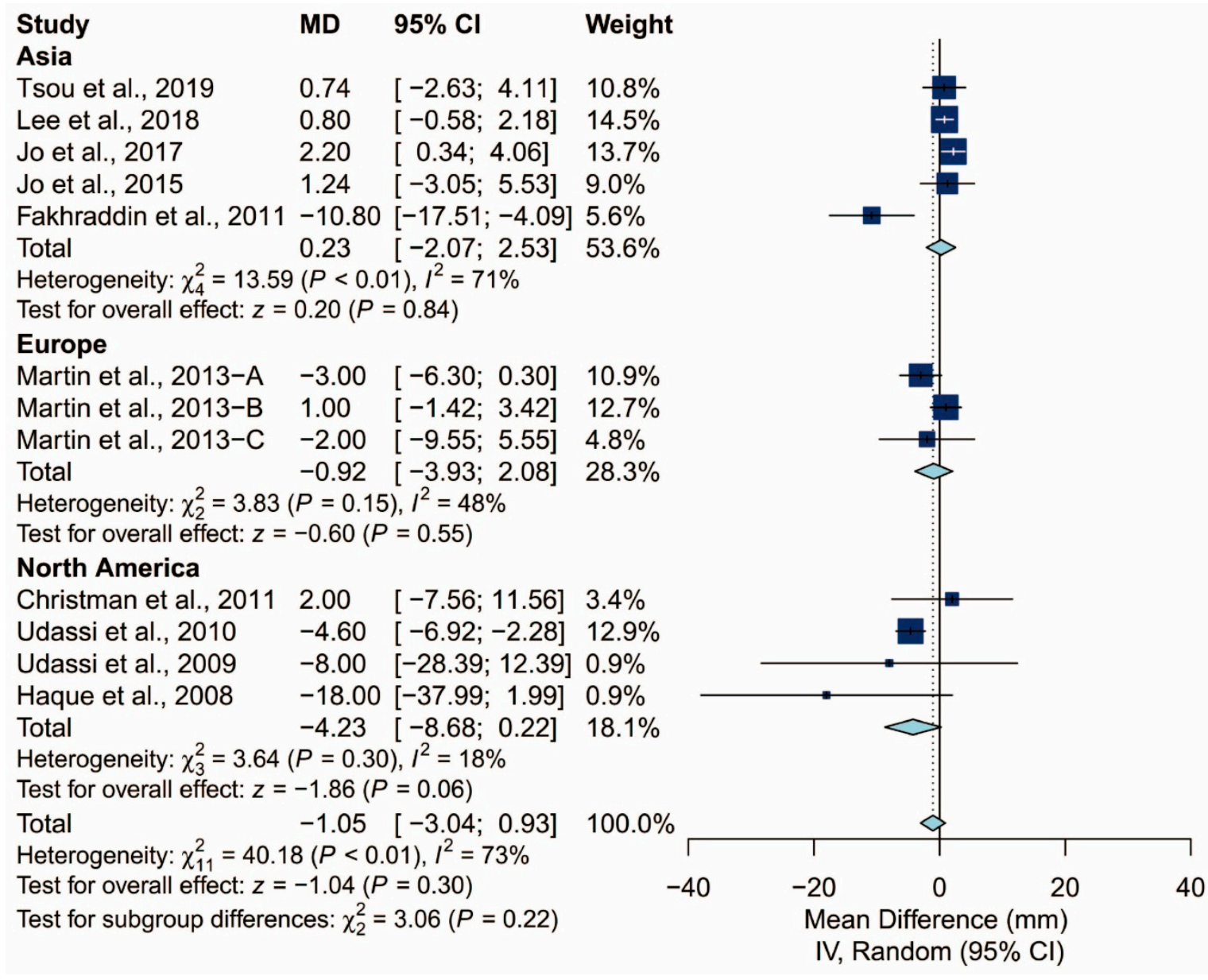

Figure 11. Subgroup analysis of chest compression rate grouped by manikin placement.

\subsection{Subgroup Analysis of Proportion of Adequate Compression Depth}

In subgroup analysis, only ventilation protocol was evaluated due to the relatively low number of included studies (Figures 12 and 13). As in the previous results, the ventilation protocol did not explain the heterogeneity observed in the proportion of adequate compression depth. Heterogeneity was high in both groups of studies: those that did not require the participants to perform ventilation and those that did $\left(\mathrm{I}^{2}=81 \%\right.$ and $\mathrm{I}^{2}=92 \%$, respectively). 


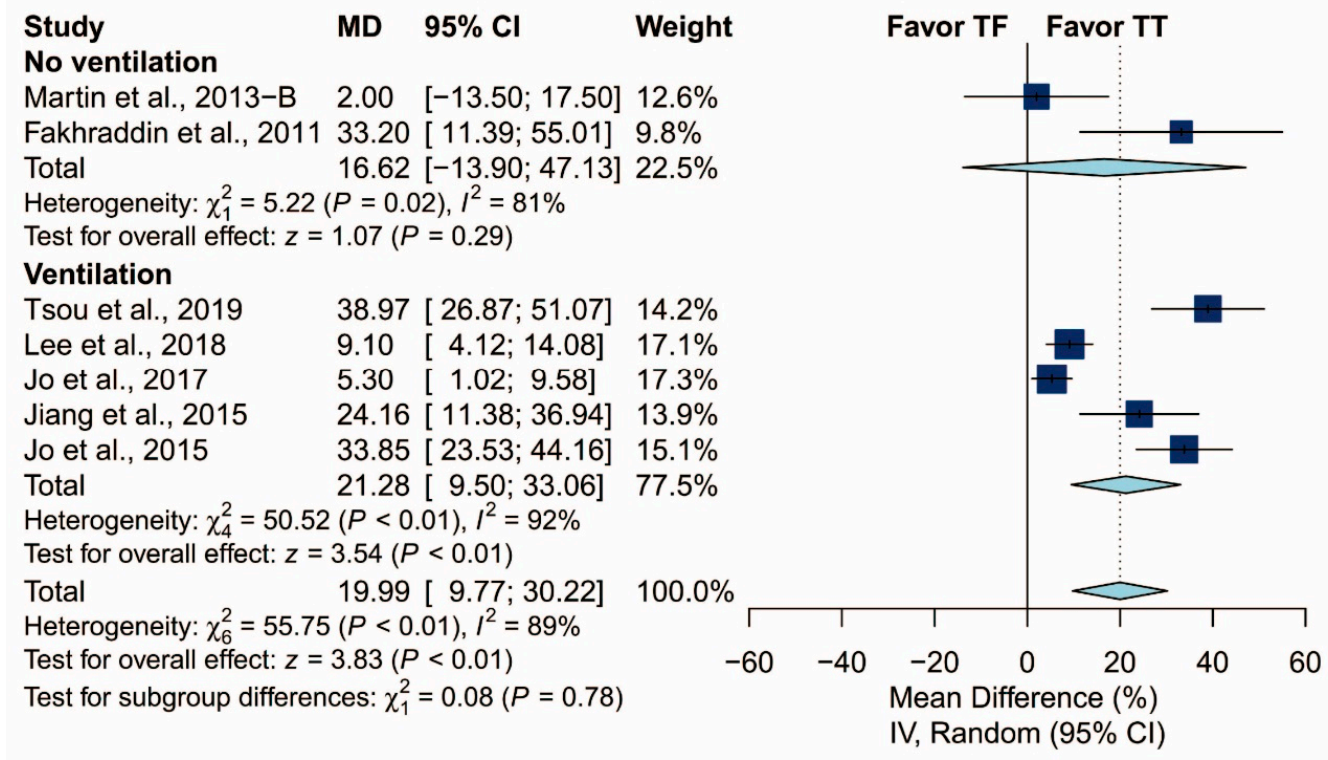

Figure 12. Subgroup analysis of the proportion of adequate compression depth grouped by ventilation protocol (yes/no).

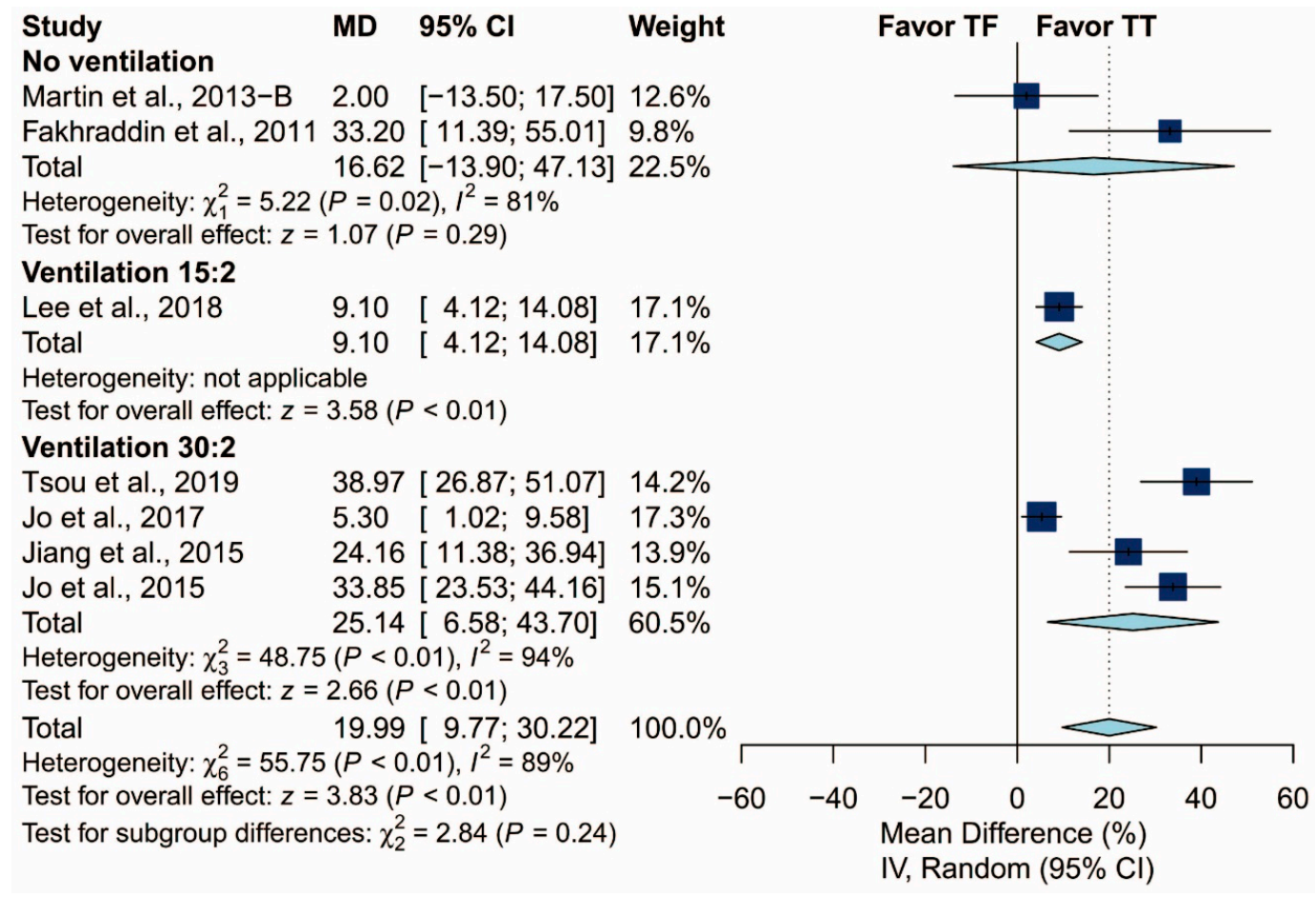

Figure 13. Subgroup analysis of the proportion of adequate compression depth grouped by ventilation protocol (15:2/30:2/no).

\subsection{Sensitivity Analysis of Chest Compression Rate}

First, leave-one-out analysis revealed that all the pooled estimates after omitting one study at a time still lie within the $95 \%$ confidence interval of the overall estimate (Figure 14A). Second, the lowest observed correlation of the TT and TF techniques among the other studies, which is 0.44 , was assumed for two studies $[25,26]$. Similarly, we replaced the original correlation with the highest observed, which is 0.95 , and zero and reperformed meta-analysis. The overall estimate remained nonsignificant after the correlation was replaced with the highest observed one (MD: $-0.87 / \mathrm{min} ; 95 \% \mathrm{CI}:-2.67$ to $0.93 ; p=0.35$; Figure $14 \mathrm{~B}$ ) and zero (MD: $-1.06 / \mathrm{min} ; 95 \%$ CI: -3.10 to $0.94 ; p=0.29$; Figure $14 \mathrm{C}$ ). Third, three potential outliers $[20,27,28]$ were 
identified in a similar fashion by three unsupervised learning algorithms (Figure 15A-C). The corresponding subsets including these potential outliers are shown in Figure 15D. However, because the GOSH plot remained heterogeneous (Figure 15E), we further explored the influence of each study by plotting the Baujat plot (Figure 15F). Two studies lay at the right side of the plot [24,30], and the corresponding subsets including these studies are shown in Figure 15G. We reperformed the meta-analysis after excluding the potential outliers, and the pooled estimate remained nonsignificant (MD: 0.79/min; $95 \% \mathrm{CI}:-0.28$ to 1.87 ; $p=0.15$; Figure $15 \mathrm{H})$ with low heterogeneity $\left(\mathrm{I}^{2}=0 \%\right)$.

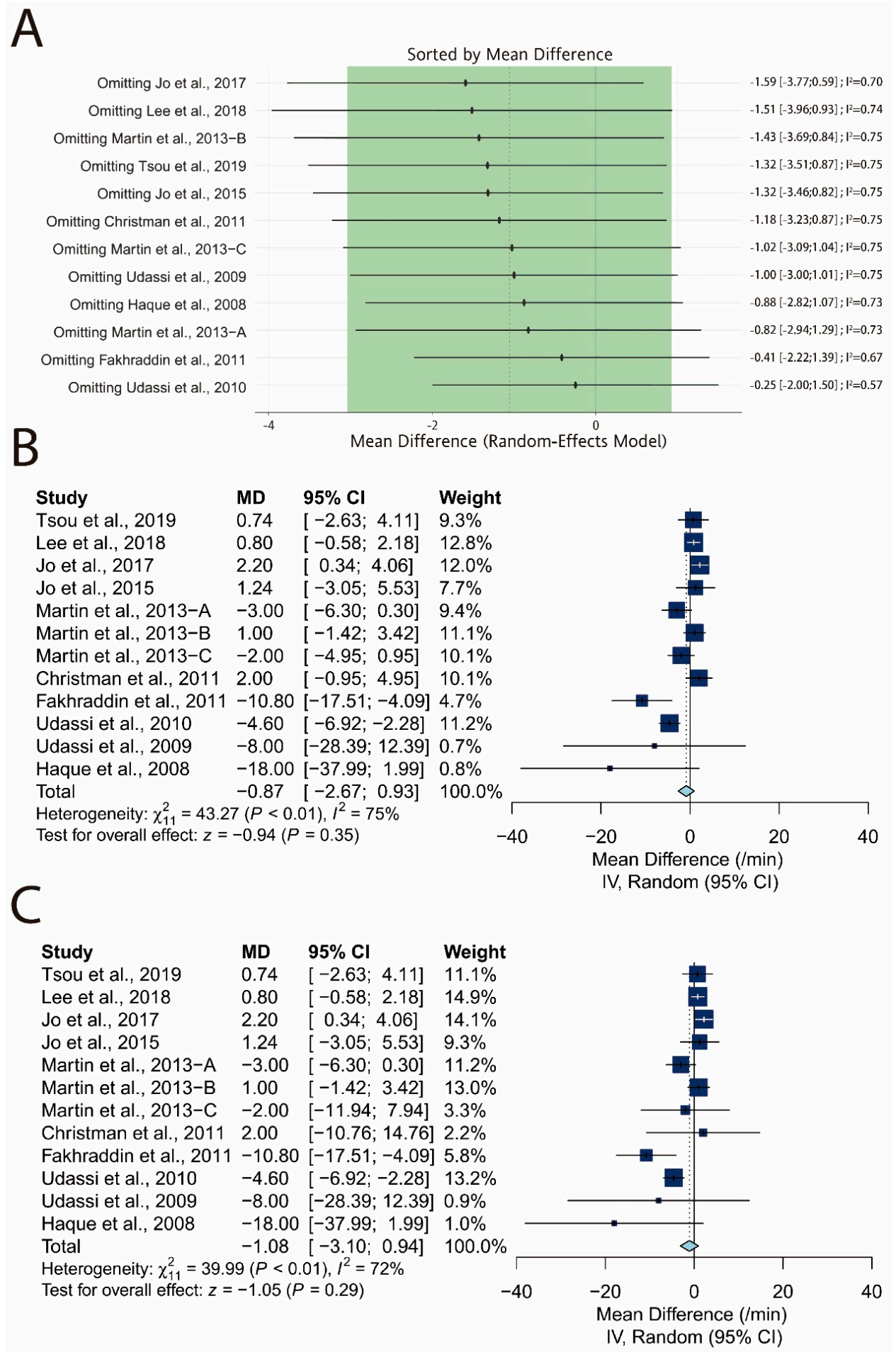

Figure 14. Sensitivity analysis of chest compression rate. (A) Leave-one-out analysis; (B) forest plot with the correlation set as the highest observed (0.95); (C) forest plot with the correlation set as 0 . 
A
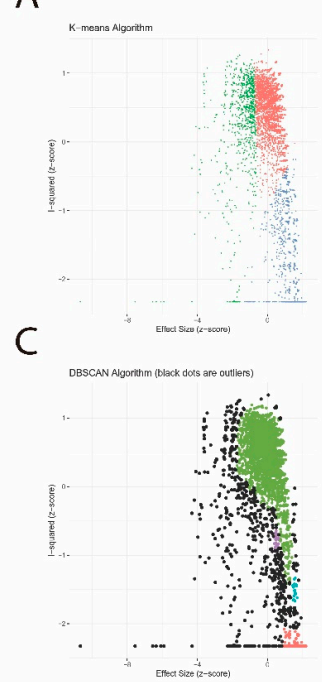

$E^{\prime}$

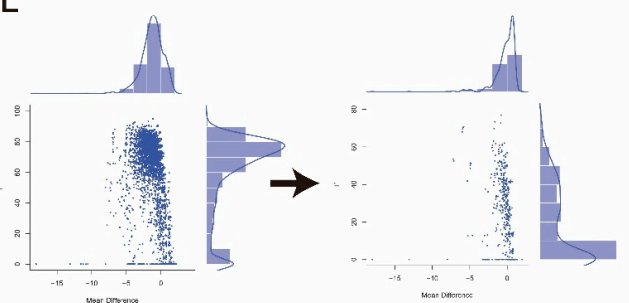

G

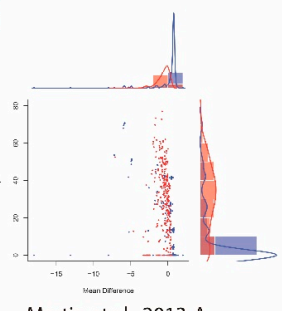

Martin et al., 2013-A

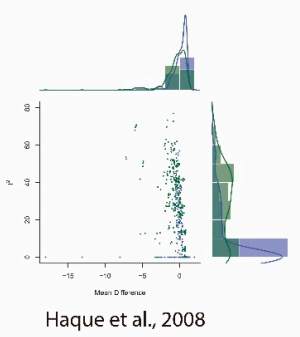

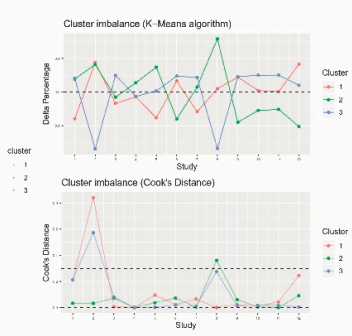

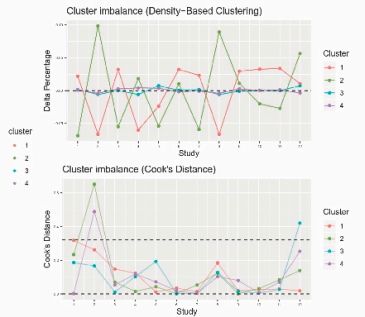

$\mathrm{F}$

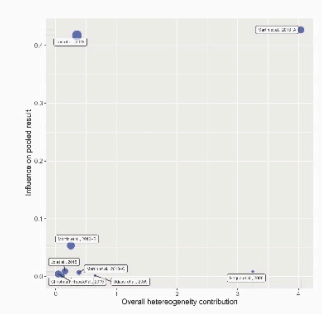

$\mathrm{H}$
B
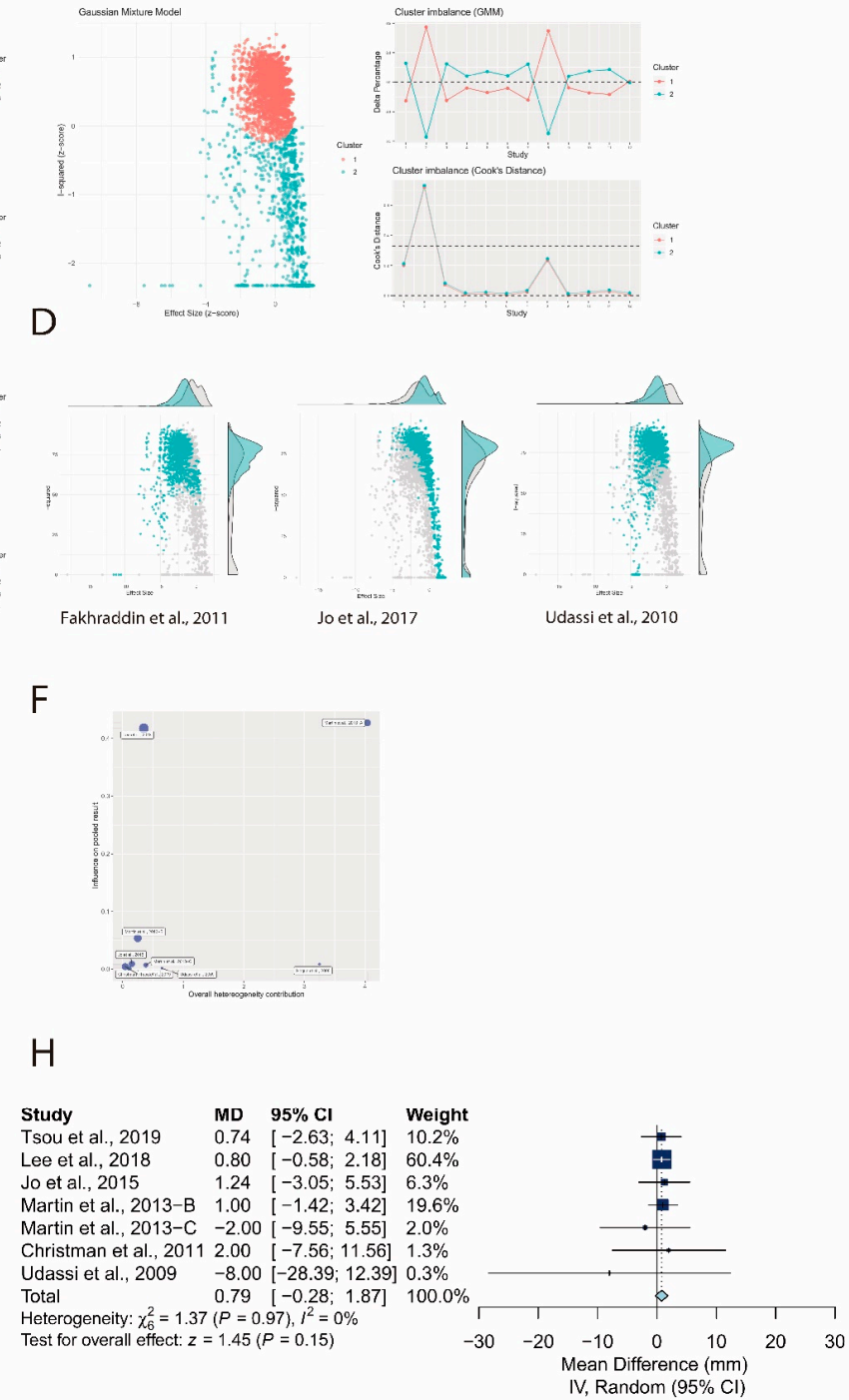

Figure 15. (A,B,C) Potential outliers identified by three unsupervised learning algorithms. (D) GOSH plots with the corresponding subsets including the potential outliers colored in green. (E) Left plot: the original GOSH plot; right plot: the GOSH plot after excluding the potential outliers in the first round. (F) Baujat plot containing the remaining studies after excluding the potential outliers in the first round. (G) GOSH plots with the corresponding subsets including the potential outliers identified from the Baujat plot colored in red and dark green. (H) Forest plot after excluding all the potential outliers.

\subsection{Leave-one-out Analysis of Proportion of Adequate Compression Depth and Hands-Off Time}

First, leave-one-out analysis revealed that all the pooled estimates after omitting one study at a time still lie within the $95 \%$ confidence interval of the overall estimate (Figure 16A). Second, the Baujat plot showed two studies located at the right side of the plot [18,21] (Figure 16B). The corresponding subsets including these potential outliers were shown in Figure 16D. However, the GOSH plot remained heterogeneous after excluding the potential outliers (Figure 16C). We reperformed the meta-analysis after excluding the potential outliers, and the pooled estimate remained significant (MD: $11.51 \%$; 95\% CI: 4.26 to 18.75; $p<0.01$; Figure 16E). In hands-off time, leave-one-out analysis was also performed for these outcomes. The results revealed that all the pooled estimates after omitting one study at a time still lie within the $95 \%$ confidence interval of the overall estimate in hands-off time. 


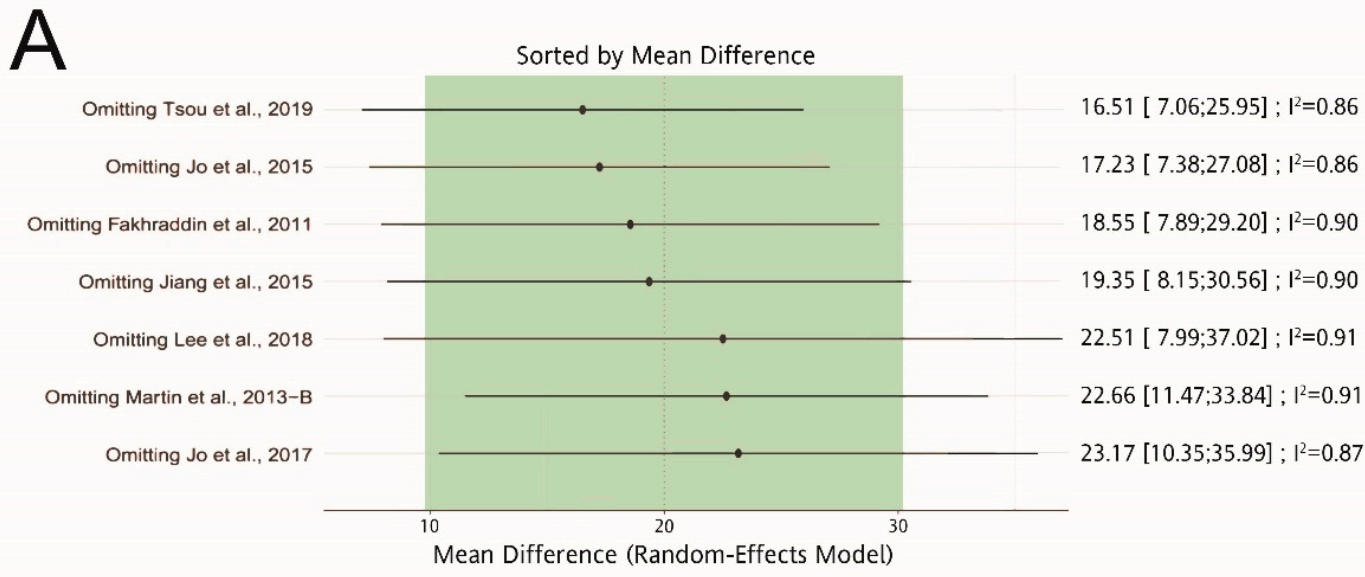

B
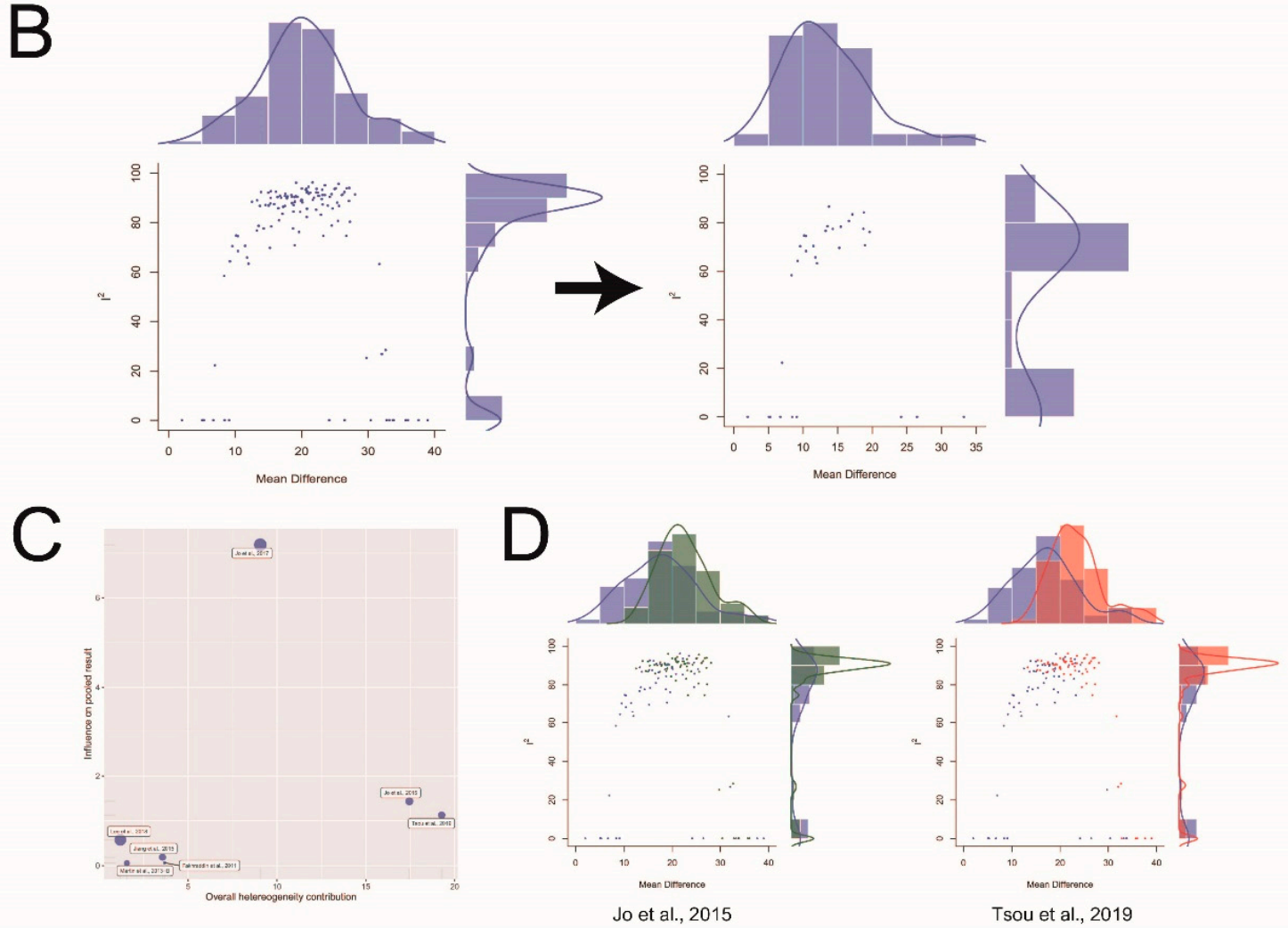

$\mathrm{E}$

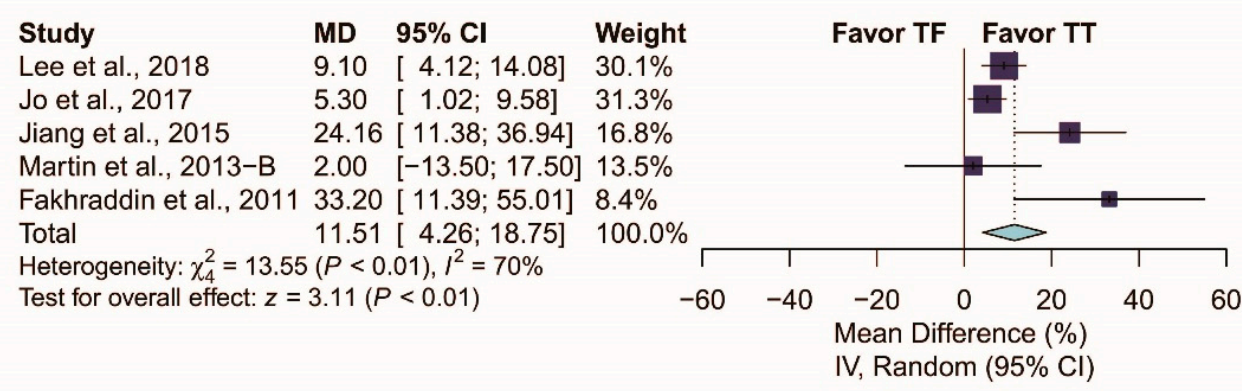

Figure 16. Sensitivity analysis of proportion of adequate compression depth. (A) Leave-one-out analysis. (B) The Baujat plot. (C) Left plot: the original GOSH plot; right plot: the GOSH plot after excluding the potential outliers identified from the Baujat plot. (D) GOSH plots with the corresponding subsets including the potential outliers identified from the Baujat plot colored in red and dark green. (E) Forest plot after excluding all the potential outliers. 


\section{Discussion}

In our meta-analysis, the results showed that the TT technique generates significantly higher proportions of adequate compression depth than the TF technique. Our data were similar to those found in the previous studies. In Michael G. Millin et al.'s study [31], the TT technique showed greater compression depth and $36.91 \%$ more adequate compression depth than the TF technique. The compression depth is not only greater but also more consistent with the TT techniques. In addition, the subgroup analysis showed that the portion of adequate compression depth is better in TT than TF, regardless of the ventilation protocol (15:2 or 30:2). These results may be explained by the fact that the TF technique was relatively unsteady and more easily caused fatigue during CPR, especially in shifting between ventilation and chest compression.

Three major challenges have been emphasized in the current guidelines for HP-CPR: shallow chest compressions, excessive compression rates, and prolonged duty cycles. In Haque et al.'s study [30], the authors revealed a trend of higher compression rate in the infant-sized manikin as compared with the adult manikins due to smaller compression displacement required. Excessive compression rates prohibited the chest wall from complete recoil, leading to decreased venous return and cardiac output. In our results, there was no significant difference in compression rates between the TT and TF techniques. This result remained the same in different manikin models and under different ventilation protocols (i.e., a C: $\mathrm{V}$ ratio of 15:2, a C:V ratio of 30:2, or no ventilation at all) in our subgroup analysis.

Hands-off time is another concern when performing the TT technique during infant CPR by a single rescuer. Although the present study suggested that there was no significant difference in hands-off time between the two techniques, the relatively low number of studies included in this outcome decreased our confidence to make such a conclusion. While most of the studies reported longer hands-off time in the TT technique [19,32-34], several modified techniques for infant CPR have been proposed to improve hands-off time and maintain adequate compression depth. In Jo et al.'s studies [20,21], they proposed that the rescuers performed the TT technique at the head of the manikin (and hence the name over-the-head two-thumb encircling technique (OTTT)). Moving the rescuer from the side to the head position of the manikin can shorten the time between chest compression and ventilation. The authors revealed that the mean hands-off time of the OTTT technique was similar to that of the TF technique ( $7.6 \pm 1.1$ vs. $7.9 \pm 1.3 \mathrm{~s}, p=0.885)$ [20]. In Jacek Smereka et al.'s studies [34-39], a new two-thumb chest compression technique (nTTT) was promoted, which consisted of the two thumbs directed at the angle of $90^{\circ}$ to the chest. This method may provide the same chest compression force as TT and get the same full recoil and hand-off time as TF. In these studies, the performance of nTTT is comparable to the recommendations laid out in the current guidelines in terms of compression depth, hands-off time, and ventilation quality. On the other hand, the "knocking-fingers" chest compression technique (KF) proposed by Jung et al. [33] is a novel chest compression technique that uses the tip of the thumb against the palmar side of the index finger with flexion of the proximal interphalangeal joint and the distal interphalangeal joint. The KF technique shortened the total hands-off time (median: $70 \mathrm{vs} .72 \mathrm{~s}$ ) while maintaining the proportion of adequate compression depth as compared with the TF technique. Further investigation is required to confirm the effects of the novel chest compression techniques.

Several limitations were noted in this study. First, although the proportion of adequate compression depth is better in TT than TF, the results may not effectively reflect the coronary perfusion pressure, which is the most effective parameter for chest compression quality. Second, although there was no significant difference in compression rates between the TT and TF techniques, it may be explained that the CPR duration was shorter than in real infant cardiac arrest. The fatigue in the CPR rescuer would be more significantly detected in the TT and TF techniques during a prolonged CPR course. However, the longest CPR duration is only five minutes in our included articles. Third, all the included studies were tested on a manikin model. Finally, there are few studies focused on our outcomes of interest, and there are especially few studies focused on hands-off time. In the future, large randomized clinical trials are necessary to confirm our results. 


\section{Conclusions}

In conclusion, our results indicate that the TT technique is superior to the TF technique in terms of adequate compression depth, without significant difference in compression rate and hands-off time.

Author Contributions: Conceptualization, C.-Y.C. and M.-Y.W.; data curation, C.-Y.C.; formal analysis, C.-Y.C., Y.-J.C., and M.-Y.W.; funding acquisition, Y.-T.H., P.-C.L., and C.-S.C.; investigation, C.-Y.C., Y.-J.C., and Y.-L.C.; methodology, Y.-T.H.; software, Y.-T.H.; validation, Y.-J.C.; visualization, C.-Y.C., Y.-L.C., C.-S.C., and M.-Y.W.; writing-original draft, C.-Y.C., Y.-T.H., Y.-J.C., Y.-L.C., P.-C.L., and M.-Y.W.; writing-review \& editing, C.-Y.C., Y.-T.H., and M.-Y.W. All authors have read and agreed to the published version of the manuscript.

Funding: This study was supported by the grant of Taipei Tzu Chi Hospital (TCRD-TPE-109-02, TCRD-TPE-109-03, TCRD-TPE-109-13).

Conflicts of Interest: The authors declare no conflict of interest.

\section{References}

1. Atkins, D.L.S.; Everson-Stewart, G.K.; Sears, M.; Daya, M.H.; Osmond, C.R.; Warden, R.A. Berg. Epidemiology and Outcomes from out-of-Hospital Cardiac Arrest in Children: The Resuscitation Outcomes Consortium Epistry-Cardiac Arrest. Circulation 2009, 119, 1484-1491. [CrossRef]

2. Young, K.D.; Gausche-Hill, M.; McClung, C.D.; Lewis, R.J. A Prospective, Population-Based Study of the Epidemiology and Outcome of out-of-Hospital Pediatric Cardiopulmonary Arrest. Pediatrics 2004, 114, 157-164. [CrossRef] [PubMed]

3. Tress, E.E.; Kochanek, P.M.; Saladino, R.A.; Manole, M.D. Cardiac Arrest in Children. J. Emerg. Trauma Shock 2010, 3, 267-272. [CrossRef] [PubMed]

4. Atkins, D.L.; Berger, S.; Duff, J.P.; Gonzales, J.C.; Hunt, E.A.; Joyner, B.L.; Meaney, P.A.; Niles, D.E.; Samson, R.A.; Schexnayder, S.M. Part 11: Pediatric Basic Life Support and Cardiopulmonary Resuscitation Quality: 2015 American Heart Association Guidelines Update for Cardiopulmonary Resuscitation and Emergency Cardiovascular Care. Circulation 2015, 132, S519-S525. [CrossRef] [PubMed]

5. Maconochie, I.K.; De Caen, A.R.; Aickin, R.; Atkins, D.L.; Biarent, D.; Guerguerian, A.M.; Kleinman, D.A.; Kloeck, M.E.; Meaney, P.A.; Nadkarni, V.M.; et al. Part 6: Pediatric Basic Life Support and Pediatric Advanced Life Support: 2015 International Consensus on Cardiopulmonary Resuscitation and Emergency Cardiovascular Care Science with Treatment Recommendations. Circulation 2015, 95, E147-E168.

6. Houri, P.K.; Frank, L.R.; Menegazzi, J.J.; Taylor, R. A Randomized, Controlled Trial of Two-Thumb Vs Two-Finger Chest Compression in a Swine Infant Model of Cardiac Arrest [See Comment]. Prehosp. Emerg. Care 1997, 1, 65-67. [CrossRef]

7. Menegazzi, J.J.; Auble, T.E.; Nicklas, K.A.; Hosack, G.M.; Rack, L.; Goode, J.S. Two-Thumb Versus Two-Finger Chest Compression During CRP in a Swine Infant Model of Cardiac Arrest. Ann. Emerg. Med. 1993, 22, 240-243. [CrossRef]

8. Whitelaw, C.C.; Slywka, B.; Goldsmith, L.J. Comparison of a Two-Finger Versus Two-Thumb Method for Chest Compressions by Healthcare Providers in an Infant Mechanical Model. Resuscitation 2000, 43, 213-216. [CrossRef]

9. Dorfsman, M.L.; Menegazzi, J.J.; Wadas, R.J.; Auble, T.E. Two-Thumb Vs. Two-Finger Chest Compression in an Infant Model of Prolonged Cardiopulmonary Resuscitation. Acad. Emerg. Med. 2000, 7, 1077-1082. [CrossRef]

10. Moher, D.; Liberati, A.; Tetzlaff, J.; Altman, D.G. Prisma Group. Preferred Reporting Items for Systematic Reviews and Meta-Analyses: The Prisma Statement. PLoS Med. 2009, 6, e1000097. [CrossRef]

11. Sterne, J.A.C.; Savović, J.; Page, M.J.; Elbers, R.G.; Blencowe, N.S.; Boutron, I.; Cates, C.J.; Cheng, H.-Y.; Corbett, M.S.; Eldridge, S.M.; et al. Rob 2: A Revised Tool for Assessing Risk of Bias in Randomised Trials. BMJ 2019, 366, 14898. [CrossRef] [PubMed]

12. DerSimonian, R.; Laird, N. Meta-Analysis in Clinical Trials. Control. Clin. Trials 1986, 7, 177-188. [CrossRef]

13. Olkin, I.; Dahabreh, L.J.; Trikalinos, T.A. Gosh—A Graphical Display of Study Heterogeneity. Res. Synth. Methods 2012, 3, 214-223. [CrossRef]

14. Hartigan, J.A.; Wong, M.A. Algorithm as 136: A K-Means Clustering Algorithm. J. R. Stat. Soc. Ser. C Appl. Stat. 1979, 28, 100-108. [CrossRef] 
15. Schubert, E.; Sander, J.; Ester, M.; Kriegel, H.P.; Xu, X. DBSCAN Revisited, Revisited: Why and How You Should (Still) Use DBSCAN. ACM Transactions on Database Systems. TODS 2017, 42, 3. [CrossRef]

16. Leisch, F. Flexmix: A General Framework for Finite Mixture Models and Latent Glass Regression in R. J. Stat. Software 2004, 11, 1-18. [CrossRef]

17. Baujat, B.; Mahe, C.; Pignon, J.P.; Hill, C. A Graphical Method for Exploring Heterogeneity in Meta-Analyses: Application to a Meta-Analysis of 65 Trials. Stat. Med. 2002, 21, 2641-2652. [CrossRef]

18. Tsou, J.Y.; Kao, C.L.; Chang, C.J.; Tu, Y.F.; Su, F.C.; Chi, C.H. Biomechanics of Two-Thumb Versus Two-Finger Chest Compression for Cardiopulmonary Resuscitation in an Infant Manikin Model. Eur. J. Emerg. Med. 2020, 27, 132-136. [CrossRef]

19. Lee, S.Y.; Hong, J.Y.; Oh, J.H.; Son, S.H. The Superiority of the Two-Thumb over the Two-Finger Technique for Single-Rescuer Infant Cardiopulmonary Resuscitation. Eur. J. Emerg. Med. 2018, 25, 372-376. [CrossRef]

20. Jo, C.H.; Cho, G.C.; Lee, C.H. Two-Thumb Encircling Technique over the Head of Patients in the Setting of Lone Rescuer Infant CPR Occurred During Ambulance Transfer: A Crossover Simulation Study. Pediatr. Emerg. Care 2017, 33, 462-466. [CrossRef]

21. Jo, C.H.; Jung, H.S.; Cho, G.C.; Oh, Y.J. Over-the-Head Two-Thumb Encircling Technique as an Alternative to the Two-Finger Technique in the in-Hospital Infant Cardiac Arrest Setting: A Randomised Crossover Simulation Study. Emerg. Med. J. 2015, 32, 703-707. [CrossRef] [PubMed]

22. Jiang, J.; Zou, Y.; Shi, W.; Zhu, Y.; Tao, R.; Jiang, Y.; Lu, Y.; Tong, J. Two-Thumb-Encircling Hands Technique Is More Advisable Than 2-Finger Technique When Lone Rescuer Performs Cardiopulmonary Resuscitation on Infant Manikin. Am. J. Emerg. Med. 2015, 33, 531-534. [CrossRef]

23. Martin, P.S.; Kemp, A.M.; Theobald, P.S.; Maguire, S.A.; Jones, M.D. Does a More "Physiological" Infant Manikin Design Effect Chest Compression Quality and Create a Potential for Thoracic Over-Compression During Simulated Infant CPR? Resuscitation 2013, 84, 666-671. [CrossRef]

24. Martin, P.S.; Kemp, A.M.; Theobald, P.S.; Maguire, S.A.; Jones, M.D. Do Chest Compressions During Simulated Infant CPR Comply with International Recommendations? Arch. Dis Child. 2013, 98, 576-581. [CrossRef] [PubMed]

25. Martin, P.; Theobald, P.; Kemp, A.; Maguire, S.; Maconochie, I.; Jones, M. Real-Time Feedback Can Improve Infant Manikin Cardiopulmonary Resuscitation by up to 79\%-A Randomised Controlled Trial. Resuscitation 2013, 84, 1125-1130. [CrossRef] [PubMed]

26. Christman, C.; Hemway, R.J.; Wyckoff, M.H.; Perlman, J.M. The Two-Thumb Is Superior to the Two-Finger Method for Administering Chest Compressions in a Manikin Model of Neonatal Resuscitation. Arch. Dis. Child. Fetal Neonatal. Ed. 2011, 96, F99-F101. [CrossRef]

27. Udassi, S.; Udassi, J.P.; Lamb, M.A.; Theriaque, D.W.; Shuster, J.J.; Zaritsky, A.L.; Haque, L.U. Two-Thumb Technique Is Superior to Two-Finger Technique During Lone Rescuer Infant Manikin CPR. Resuscitation 2010, 81, 712-717. [CrossRef]

28. Fakhraddin, B.Z.; Shimizu, N.; Kurosawa, S.; Sakai, H.; Miyasaka, K.; Mizutani, S. New Method of Chest Compression for Infants in a Single Rescuer Situation: Thumb-Index Finger Technique. J. Med. Dent. Sci. 2011, 58, 15-22.

29. Udassi, S.; Theriaque, D.W.; Shuster, J.J.; Zaritsky, A.L.; Udassi, J.P.; Haque, I.U. Effect of Alternative Chest Compression Techniques in Infant and Child on Rescuer Performance. Pediatr. Crit. Care Med. 2009, 10, 328-333. [CrossRef]

30. Haque, I.U.; Udassi, J.P.; Udassi, S.; Theriaque, D.W.; Shuster, J.J.; Zaritsky, A.L. Chest Compression Quality and Rescuer Fatigue with Increased Compression to Ventilation Ratio During Single Rescuer Pediatric CPR. Resuscitation 2008, 79, 82-89. [CrossRef]

31. Millin, M.G.; Bogumil, D.; Fishe, J.N.; Burke, R.V. Comparing the Two-Finger Versus Two-Thumb Technique for Single Person Infant CPR: A Systematic Review and Meta-Analysis. Resuscitation 2020, 148, 161-172. [CrossRef] [PubMed]

32. Pellegrino, J.L.; Bogumil, D.; Epstein, J.L.; Burke, R.V. Two-Thumb-Encircling Advantageous for Lay Responder Infant CPR: A Randomised Manikin Study. Arch. Dis. Child. 2019, 104, 530-534. [CrossRef] [PubMed]

33. Jung, W.J.; Hwang, S.O.; Kim, H.I.; Cha, Y.S.; Kim, O.H.; Kim, H.; Lee, K.H.; Cha, K.C. Knocking-Fingers' Chest Compression Technique in Infant Cardiac Arrest: Single-Rescuer Manikin Study. Eur. J. Emerg. Med. 2019, 26, 261-265. [CrossRef] [PubMed] 
34. Smereka, J.; Szarpak, L.; Smereka, A.; Leung, S.; Ruetzler, K. Evaluation of New Two-Thumb Chest Compression Technique for Infant CPR Performed by Novice Physicians. A Randomized, Crossover, Manikin Trial. Am. J. Emerg. Med. 2017, 35, 604-609. [CrossRef] [PubMed]

35. Smereka, J.; Bielski, K.; Ladny, J.R.; Ruetzler, K.; Szarpak, L. Evaluation of a Newly Developed Infant Chest Compression Technique: A Randomized Crossover Manikin Trial. Medicine 2017, 96, e5915. [CrossRef] [PubMed]

36. Smereka, J.; Kasiński, M.; Smereka, A.; Ładny, J.R.; Szarpak, L. The Quality of a Newly Developed Infant Chest Compression Method Applied by Paramedics: A Randomised Crossover Manikin Trial. Kardiol. Pol. 2017, 75, 589-595. [CrossRef] [PubMed]

37. Smereka, J.; Madziala, M.; Szarpak, L. Comparison of Two Infant Chest Compression Techniques During Simulated Newborn Cardiopulmonary Resuscitation Performed by a Single Rescuer: A Randomized, Crossover Multicenter Trial. Cardiol. J. 2019, 26, 761-768. [CrossRef]

38. Smereka, J.; Szarpak, L.; Ladny, J.R.; Rodriguez-Nunez, A.; Ruetzler, K. A Novel Method of Newborn Chest Compression: A Randomized Crossover Simulation Study. Front. Pediatr. 2018, 6, 159. [CrossRef]

39. Smereka, J.; Szarpak, L.; Rodríguez-Núñez, A.; Ladny, J.R.; Leung, S.; Ruetzler, K. A Randomized Comparison of Three Chest Compression Techniques and Associated Hemodynamic Effect During Infant CPR: A Randomized Manikin Study. Am. J. Emerg. Med. 2017, 35, 1420-1425. [CrossRef]

(C) 2020 by the authors. Licensee MDPI, Basel, Switzerland. This article is an open access article distributed under the terms and conditions of the Creative Commons Attribution (CC BY) license (http://creativecommons.org/licenses/by/4.0/). 